\title{
Minkowski Valuations and Generalized Valuations
}

\author{
Franz E. Schuster and Thomas Wannerer
}

\begin{abstract}
A convolution representation of continuous translation invariant and $\mathrm{SO}(n)$ equivariant Minkowski valuations is established. This is based on a new classification of translation invariant generalized spherical valuations. As applications, Crofton and kinematic formulas for Minkowski valuations are obtained.
\end{abstract}

\section{Introduction}

A valuation on convex bodies (non-empty compact convex sets) is a finitely additive function. More precisely, let $\mathcal{K}^{n}$ denote the space of convex bodies in $\mathbb{R}^{n}$ endowed with the Hausdorff metric. A map $\phi: \mathcal{K}^{n} \rightarrow \mathcal{A}$ with values in an Abelian semigroup $\mathcal{A}$ is a valuation if

$$
\phi(K)+\phi(L)=\phi(K \cup L)+\phi(K \cap L)
$$

whenever $K \cup L$ is convex. As a generalization of the notion of measure and as the crucial ingredient in Dehn's solution of Hilbert's third problem, scalar valuations (where $\mathcal{A}=\mathbb{R}$ or $\mathbb{C}$ ) have long played a central role in convex and discrete geometry (see [39] or [56, Chapter 6]). The most famous classical result on valuations is a celebrated characterization of rigid motion invariant valuations by Hadwiger [33] (which was slightly improved later by Klain [37]).

Theorem 1 ([33, 37]) The intrinsic volumes $V_{0}, V_{1}, \ldots, V_{n}$ form a basis of the vector space of all continuous scalar valuations on $\mathcal{K}^{n}$ which are translation and $\mathrm{SO}(n)$ invariant.

Hadwiger's characterization theorem had a transformative effect on integral geometry. It not only allowed for almost effortless proofs of the principal and more general kinematic formulas (see, e.g., [39]) but also made the importance of precise descriptions of classes of invariant valuations evident. Still to this day, Theorem 1 often serves as a starting point for the classification of invariant scalar valuations (see, e.g., [4, 9, 13, 31, 43]) and, more general, equivariant tensor valued valuations, where $\mathcal{A}=\operatorname{Sym}^{k} \mathbb{R}^{n}$ (see [3, 10, 35, 67]). These results in turn were critical for the tremendous progress in integral geometry of recent years (see [5, 16, 17, 20, 35, 66] and the references therein).

In 1974 Schneider [54, 55] first investigated valuations, where $\mathcal{A}=\mathcal{K}^{n}$ and addition on $\mathcal{K}^{n}$ is the usual Minkowski addition. In a more recent influential article, Ludwig [41] coined the name Minkowski valuations for such maps and started a line of research concerned with the classification of Minkowski valuations intertwining linear transformations, see [1, 2, 30, 40, 42, 61, 65]. 
The recent results on Minkowski valuations which are equi- or contravariant with respect to linear transformations show that they often form convex cones generated by finitely many valuations, such as the projection or difference body operators. In contrast to this, the cone of translation invariant and $\mathrm{SO}(n)$ equivariant Minkowski valuations is infinite dimensional. This is one reason why no full analogue of Theorem 1 for Minkowski valuations has been obtained yet, except for dimension $n=2$, where Schneider [55] already established such a result. We therefore assume throughout that $n \geq 3$.

About a decade ago, Kiderlen [36] and the first author [59] were the first to obtain convolution representations of translation invariant and $\mathrm{SO}(n)$ equivariant continuous Minkowski valuations. However, their results were limited to the case of valuations of degree 1 and $n-1$, respectively, where a map $\Phi$ from $\mathcal{K}^{n}$ to $\mathcal{K}^{n}$ (or $\mathbb{R}$ ) is said to have degree $i$ if $\Phi(\lambda K)=\lambda^{i} \Phi K$ for $K \in \mathcal{K}^{n}$ and $\lambda>0$. The convolution of functions and measures on $\mathbb{S}^{n-1}$ used in [36] and [59] is induced from the group $\mathrm{SO}(n)$ by identifying $\mathbb{S}^{n-1}$ with the homogeneous space $\mathrm{SO}(n) / \mathrm{SO}(n-1)$ (see Section 2 for details).

Under additional smoothness assumptions, the first author in [60] and jointly with the second author in [62 extended the results from [36] and [59] to the remaining (non-trivial) degrees $i \in\{2, \ldots, n-2\}$ when the Minkowski valuations are even. (McMullen [47] showed that only integer degrees $0 \leq i \leq n$ can occur.) However, the techniques employed in 60 6 or $6 \mathbf{6 2}$ were not suited to describe merely continuous Minkowski valuations, which is the goal since the 1970s.

In this article we establish a precise description of all continuous translation invariant and $\mathrm{SO}(n)$ equivariant Minkowski valuations without any further assumptions on the parity or degree of the valuations. As we explain in Section 5, our main theorem generalizes and implies all previously obtained convolution representations of Minkowski valuations intertwining rigid motions. In order to state our result, recall that a convex body $K \in \mathcal{K}^{n}$ is uniquely determined by its support function $h_{K}(u)=\max \{u \cdot x: x \in K\}$ for $u \in \mathbb{S}^{n-1}$ and let $\mathcal{M}_{\mathrm{o}}\left(\mathbb{S}^{n-1}\right)$ and $C_{\mathrm{o}}\left(\mathbb{S}^{n-1}\right)$ denote the spaces of signed Borel measures and continuous functions on $\mathbb{S}^{n-1}$, respectively, having their center of mass at the origin.

Theorem 2 If $\Phi: \mathcal{K}^{n} \rightarrow \mathcal{K}^{n}$ is a continuous Minkowski valuation which is translation invariant and $\mathrm{SO}(n)$ equivariant, then there exist uniquely determined $c_{0}, c_{n} \geq 0, \mathrm{SO}(n-1)$ invariant $\mu_{i} \in \mathcal{M}_{\mathrm{o}}\left(\mathbb{S}^{n-1}\right), 1 \leq i \leq n-2$, and an $\mathrm{SO}(n-1)$ invariant $f_{n-1} \in C_{\mathrm{o}}\left(\mathbb{S}^{n-1}\right)$ such that

$$
h_{\Phi K}=c_{0}+\sum_{i=1}^{n-2} S_{i}(K, \cdot) * \mu_{i}+S_{n-1}(K, \cdot) * f_{n-1}+c_{n} V_{n}(K)
$$

for every $K \in \mathcal{K}^{n}$. 
The Borel measures $S_{i}(K, \cdot), 1 \leq i \leq n-1$, on $\mathbb{S}^{n-1}$ are Aleksandrov's area measures (see, e.g., [56]) associated with $K \in \mathcal{K}^{n}$. If $K$ is sufficiently smooth and has positive curvature, then each $S_{i}(K, \cdot)$ is absolutely continuous with respect to spherical Lebesgue measure and its density is (up to a constant) given by the $i$ th elementary symmetric function of the principal radii of curvature of $K$.

The equality in (1.1) has to be understood in the sense of measures, where we identify $h \in C\left(\mathbb{S}^{n-1}\right)$ with the absolutely continuous measure with density $h$. For $n \leq 4$, we show in Section 5 that if $\Phi$ has degree 1 or 2, then the measures $\mu_{1}$ or $\mu_{2}$, respectively, are in fact absolutely continuous with a density in $L^{2}\left(\mathbb{S}^{n-1}\right)$. However, this is no longer true in general when $n>4$.

The proof of Theorem 2 is based on new techniques involving translation invariant generalized valuations which were only recently introduced by Alesker and Faifman [9] (see also [15]). Generalized valuations are related to smooth valuations in the same way that distributions are related to smooth functions. More precisely, let $\mathbf{V a l}_{i}^{\infty}, 0 \leq i \leq n$, denote the space of smooth translation invariant valuations of degree $i$ endowed with the Gårding topology which makes it a Fréchet space (see Section 3 for more information). The space $\mathbf{V a l}_{i}^{-\infty}$ of (translation invariant) generalized valuations of degree $i$ is defined as the topological dual

$$
\operatorname{Val}_{i}^{-\infty}:=\left(\mathbf{V a l}_{n-i}^{\infty}\right)^{*}
$$

endowed with the weak topology.

As part of his far reaching reconceptualization of integral geometry, Alesker [6] discovered a continuous non-degenerate bilinear pairing

$$
\langle\cdot, \cdot\rangle: \mathrm{Val}_{i}^{\infty} \times \mathrm{Val}_{n-i}^{\infty} \rightarrow \mathbb{R}
$$

for $0 \leq i \leq n$ (see also Section 3). The induced Poincaré duality map

$$
\operatorname{pd}: \mathbf{V a l}_{i}^{\infty} \rightarrow\left(\mathbf{V a l}_{n-i}^{\infty}\right)^{*}=\mathbf{V a l}_{i}^{-\infty}
$$

is therefore continuous, injective and has dense image with respect to the weak topology. This was the motivation for definition (1.2) and shows that $\mathrm{Val}_{i}^{-\infty}$ can be seen as a completion of $\mathbf{V a l}_{i}^{\infty}$ with respect to the weak topology. Alesker [8] also proved that the Poincaré duality map admits a unique continuous extension to the space $\mathbf{V a l}_{i}$ of continuous translation invariant valuations of degree $i$. Thus, just like smooth and continuous functions or, more general, signed Borel measures can be identified with subclasses of distributions (compare Section 2), we can use the Poincaré duality map in the following to identify the spaces $\mathbf{V a l}_{i}^{\infty}$ or $\mathbf{V a l}_{i}$, respectively, with certain dense subspaces of $\mathrm{Val}_{i}^{-\infty}$.

It was first observed in 60 that a translation invariant and $\mathrm{SO}(n)$ equivariant continuous Minkowski valuation $\Phi$ is uniquely determined by a scalar valuation $\varphi \in \mathbf{V a l}_{i}^{\mathrm{SO}(n-1)}$, the subspace of $\mathrm{SO}(n-1)$ invariant valuations in $\mathrm{Val}_{i}$. In turn, valuations in $\mathbf{V a l}_{i}^{\mathrm{SO}(n-1)}$ are spherical. 
Spherical (generalized) valuations correspond to spherical representations of $\mathrm{SO}(n)$ (see Section 3 for details). Let $\mathbf{V a l} i_{i}^{\infty, \text { sph }}$ and $\mathbf{V a l}_{i}^{-\infty, \mathrm{sph}}$ denote the subspaces of smooth and generalized spherical valuations, respectively, and let $C_{\mathrm{o}}^{-\infty}\left(\mathbb{S}^{n-1}\right)$ denote the space of distributions on $\mathbb{S}^{n-1}$ which vanish on restrictions of linear functions to $\mathbb{S}^{n-1}$. Our second main result, which is critical for the proof of Theorem 2 but also of independent interest, is the following classification of (generalized) spherical valuations.

Theorem 3 Suppose that $1 \leq i \leq n-1$.

(a) The map $\mathrm{E}_{i}: C_{\mathrm{o}}^{\infty}\left(\mathbb{S}^{n-1}\right) \rightarrow \mathbf{V a l}_{i}^{\infty, \mathrm{sph}}$, defined by

$$
\left(\mathrm{E}_{i} f\right)(K)=\int_{\mathbb{S}^{n-1}} f(u) d S_{i}(K, u),
$$

is an $\mathrm{SO}(n)$ equivariant isomorphism of topological vector spaces which admits a unique extension by continuity in the weak topologies to an isomorphism

$$
\widetilde{\mathrm{E}}_{i}: C_{\mathrm{o}}^{-\infty}\left(\mathbb{S}^{n-1}\right) \rightarrow \mathrm{Val}_{i}^{-\infty, \mathrm{sph}} .
$$

(b) The space $\mathbf{V a l}_{i}^{\mathrm{SO}(n-1)}$ is contained in $\widetilde{\mathrm{E}}_{i}\left(\mathcal{M}_{\mathrm{o}}\left(\mathbb{S}^{n-1}\right)\right)$ if $i \leq n-2$ and in $\widetilde{\mathrm{E}}_{i}\left(C_{\mathrm{o}}\left(\mathbb{S}^{n-1}\right)\right)$ if $i=n-1$.

Theorem 3 (a) for $i=1$ was recently proved by Alesker, see [11, Appendix]. Theorem 3 (b) for $i=n-1$ follows from a classical result of McMullen [48].

Characterizations of Minkowski valuations, in particular, earlier versions of Theorem 2, have had far reaching implications for isoperimetric type inequalities (see, e.g., [2, 11, 32, 44-46, 61]). Motivated by a recent important Crofton type formula for the identity map of Goodey and Weil [25], we show in the final section of this paper how Theorem 2 can be applied to obtain a general Crofton formula for continuous Minkowski valuations which generalizes the result from [25] and an earlier result of this type from [62. Using our new Crofton formula and Hadwiger's general integral geometric theorem, a consequence of Theorem 1 (cf. [58, p. 173]), we can then also state a kinematic formula for Minkowski valuations.

\section{Preliminaries}

In this section we first recall basic notions from Riemannian geometry and compute several quantities in cylindrical coordinates on $\mathbb{S}^{n-1}$ which will be needed in the proof of Theorem 3 (b). Next, we collect background material from representation theory and harmonic analysis, in particular, about the convolution of functions and measures on $\mathbb{S}^{n-1}$ and its relation to the theory of spherical harmonics. We also recall some well known facts about distributions on $\mathbb{S}^{n-1}$ and the definition of Berg's functions used in the solution of the classical Christoffel problem. 
Although in this article we are mainly concerned with the Euclidean unit sphere $\mathbb{S}^{n-1}$ in $\mathbb{R}^{n}$ and the Lie group $\mathrm{SO}(n)$ of proper rotations of $\mathbb{R}^{n}$, let us first consider a general smooth manifold $M$. We denote by $C^{\infty}(M)$ the space of all smooth functions on $M$ equipped with the Fréchet space topology of uniform convergence of each finite number of derivatives on each compact subset of $M$. For a Banach space $X$, the Fréchet space $C^{\infty}(M, X)$ of all infinitely differentiable functions on $M$ with values in $X$ is defined similarly.

If in addition $M$ is compact and endowed with a Riemannian metric, then the $C^{k}$ norm $\|f\|_{C^{k}}$ of a function $f \in C^{k}(M)$ is defined by (see, e.g., [52, p. 301])

$$
\|f\|_{C^{k}}=\sum_{j=0}^{k} \max _{M}\left|\nabla^{j} f\right|
$$

where $\nabla$ denotes the covariant derivative with respect to the given Riemannian metric and $\left|\nabla^{j} f\right|$ is the (Euclidean) norm of the tensor field $\nabla^{j} f$.

Among other quantities, we compute in the following example the $C^{2}$ norm of an $\mathrm{SO}(n-1)$ invariant function on $\mathbb{S}^{n-1}$ more explicitly. This will be useful later on for the proof of Theorem 3 (b).

\section{Example 2.1}

In this article we use $\bar{e} \in \mathbb{S}^{n-1}$ to denote an arbitrary but fixed point (the pole) of the sphere and we write $\mathrm{SO}(n-1)$ for the stabilizer in $\mathrm{SO}(n)$ of $\bar{e}$. Clearly, every $u \in \mathbb{S}^{n-1} \backslash\{-\bar{e}, \bar{e}\}$ can be written uniquely in the form

$$
u=t \bar{e}+\sqrt{1-t^{2}} v
$$

for some $t \in(-1,1)$ and $v \in \mathbb{S}_{\bar{e}}^{n-2}=\left\{w \in \mathbb{S}^{n-1}: \bar{e} \cdot w=0\right\}$. In the cylindrical coordinates (2.2), the (standard) metric tensor $\varrho$ on $\mathbb{S}^{n-1}$ is given by

$$
\varrho=\frac{1}{1-t^{2}} d t \otimes d t+\left(1-t^{2}\right) d v \otimes d v
$$

where $d v \otimes d v$ is the metric tensor on $\mathbb{S}_{\bar{e}}^{n-2}$.

Let $\Delta_{\mathbb{S}}$ denote the Laplacian (or Laplace-Beltrami operator) on $\mathbb{S}^{n-1}$ and recall that, for $f, g \in C^{2}\left(\mathbb{S}^{n-1}\right)$, we have

$$
\int_{\mathbb{S}^{n-1}} f(u) \Delta_{\mathbb{S}} g(u) d u=\int_{\mathbb{S}^{n-1}} g(u) \Delta_{\mathbb{S}} f(u) d u .
$$

Using (2.3), one can easily obtain the following expression for the Laplacian in cylindrical coordinates (cf. [12, Proposition 2.6])

$$
\Delta_{\mathbb{S}}=\frac{1}{\sqrt{|\varrho|}} \partial_{i}\left(\sqrt{|\varrho|} \varrho^{i j} \partial_{j}\right)=\left(1-t^{2}\right) \frac{\partial^{2}}{\partial t^{2}}-(n-1) t \frac{\partial}{\partial t}+\frac{1}{1-t^{2}} \bar{\Delta}_{\mathbb{S}}
$$

where $\bar{\Delta}_{\mathbb{S}}$ denotes the Laplacian on $\mathbb{S}_{\bar{e}}^{n-2}$. 
Now let $f \in C^{2}\left(\mathbb{S}^{n-1}\right)$ be $\mathrm{SO}(n-1)$ invariant, that is, in the cylindrical coordinates (2.2) the function $f$ depends only on $t$. Then, by (2.5), we have

$$
\Delta_{\mathbb{S}} f=\left(1-t^{2}\right) \frac{\partial^{2} f}{\partial t^{2}}-(n-1) t \frac{\partial f}{\partial t}
$$

Moreover, a straightforward computation, using again (2.3), yields

$$
|\nabla f|^{2}=\left(1-t^{2}\right)\left(\frac{\partial f}{\partial t}\right)^{2}
$$

and

$$
\left|\nabla^{2} f\right|^{2}=(n-2)\left(t \frac{\partial f}{\partial t}\right)^{2}+\left(\left(1-t^{2}\right) \frac{\partial^{2} f}{\partial t^{2}}-t \frac{\partial f}{\partial t}\right)^{2}
$$

We turn now to representations of Lie groups. First recall that since $\mathrm{SO}(n)$ is compact all its irreducible representations are finite dimensional and that the equivalence classes of irreducible complex representations of $\mathrm{SO}(n)$ are uniquely determined by their highest weights (see, e.g., [19]). These highest weights, in turn, can be indexed by $\lfloor n / 2\rfloor$-tuples of integers $\left(\lambda_{1}, \lambda_{2}, \ldots, \lambda_{\lfloor n / 2\rfloor}\right)$ such that

$$
\begin{cases}\lambda_{1} \geq \lambda_{2} \geq \cdots \geq \lambda_{\lfloor n / 2\rfloor} \geq 0 & \text { for odd } n, \\ \lambda_{1} \geq \lambda_{2} \geq \cdots \geq \lambda_{n / 2-1} \geq\left|\lambda_{n / 2}\right| & \text { for even } n .\end{cases}
$$

A notion of particular importance for our purposes is that of smooth vectors of an infinite-dimensional representation of a Lie group.

Definition Let $\rho$ be a continuous representation of a Lie group $G$ on a Banach space $X$. An element $x \in X$ is called a smooth vector if the map $z_{x}: G \rightarrow X$, defined by $z_{x}(\vartheta)=\rho(\vartheta) x$, is infinitely differentiable. The subspace of all smooth vectors in $X$ is denoted by $X^{\infty}$.

It is well known (cf. [68, Section 4.4]) that the subspace $X^{\infty}$ is a $G$ invariant and dense subset of $X$. Moreover, the map $X^{\infty} \rightarrow C^{\infty}(G, X)$, given by $x \mapsto z_{x}$, leads to an identification of $X^{\infty}$ with a closed subspace of $C^{\infty}(G, X)$. Hence, we can endow $X^{\infty}$ with the relative topology induced by $C^{\infty}(G, X)$. This topology on $X^{\infty}$ is called the Garding topology and turns $X^{\infty}$ into a Fréchet space. An important property of the Gårding topology on $X^{\infty}$ is that the restriction of the representation of $G$ to $X^{\infty}$ is continuous.

In the following lemma, we state two more basic facts about smooth vectors which we will use frequently. 
Lemma 2.2 Let $G$ be a Lie Group.

(a) If $\rho$ and $\tau$ are continuous representations of $G$ on Banach spaces $X$ and $Y$ and $T: X \rightarrow Y$ is a continuous and $G$ equivariant linear map, then $T\left(X^{\infty}\right) \subseteq Y^{\infty}$ and the restriction $T: X^{\infty} \rightarrow Y^{\infty}$ is continuous.

(b) If $H$ is a closed subgroup of $G$, then the smooth vectors of the left regular representation of $G$ on $C(G / H)$ are precisely the smooth functions on $G / H$, that is, $(C(G / H))^{\infty}=C^{\infty}(G / H)$.

In this article, we are specifically interested in spherical representations of $\mathrm{SO}(n)$ with respect to $\mathrm{SO}(n-1)$.

Definition Let $G$ be a compact Lie group and $H$ a closed subgroup of $G$. A representation $\rho$ of $G$ on a vector space $X$ is called spherical with respect to $H$ if there exists an $H$-invariant non-zero $x \in X$, that is, $\rho(\vartheta) x=x$ for every $\vartheta \in H$.

For the following two important facts about spherical representations (see, e.g., [64, p. 17]), we consider the left regular representation of $G$ on the Hilbert space $L^{2}(G / H)$ of square-integrable functions on the homogeneous space $G / H$.

Theorem 2.3 Let $G$ be a compact Lie group and $H$ a closed subgroup of $G$.

(i) Every subrepresentation of $L^{2}(G / H)$ is spherical with respect to $H$.

(ii) Every irreducible representation of $G$ which is spherical with respect to $H$ is isomorphic to a subrepresentation of $L^{2}(G / H)$.

\section{Example 2.4}

The decomposition of $L^{2}\left(\mathbb{S}^{n-1}\right)$ into an orthogonal sum of $\mathrm{SO}(n)$ irreducible subspaces is given by

$$
L^{2}\left(\mathbb{S}^{n-1}\right)=\bigoplus_{k \in \mathbb{N}} \mathcal{H}_{k}^{n}
$$

where $\mathcal{H}_{k}^{n}$ is the space of spherical harmonics of dimension $n$ and degree $k$. It is well known that the highest weights corresponding to the spaces $\mathcal{H}_{k}^{n}$ are the $\lfloor n / 2\rfloor$-tuples $(k, 0, \ldots, 0)$. Since $\mathbb{S}^{n-1}$ is diffeomorphic to the homogeneous space $\mathrm{SO}(n) / \mathrm{SO}(n-1)$, it follows from Theorem 2.3 that every irreducible representation of $\mathrm{SO}(n)$ which is spherical with respect to $\mathrm{SO}(n-1)$ is isomorphic to one of the spaces $\mathcal{H}_{k}^{n}$ and, thus, their highest weights are of the form $(k, 0, \ldots, 0), k \in \mathbb{N}$.

For the discussion of Theorem 2 and its applications to integral geometry, we need some more background from the theory of spherical harmonics (see, e.g., [28]). Let $N(n, k)$ denote the dimension of the space $\mathcal{H}_{k}^{n}$ and recall that

$$
N(n, k)=\frac{n+2 k-2}{n+k-2}\left(\begin{array}{c}
n+k-2 \\
n-2
\end{array}\right)=\mathrm{O}\left(k^{n-2}\right) \text { as } k \rightarrow \infty .
$$


Using $\pi_{k}: L^{2}\left(\mathbb{S}^{n-1}\right) \rightarrow \mathcal{H}_{k}^{n}$ to denote the orthogonal projection, we can write

$$
f \sim \sum_{k=0}^{\infty} \pi_{k} f
$$

for the (condensed) Fourier expansion of $f \in L^{2}\left(\mathbb{S}^{n-1}\right)$. Recall that the Fourier series in (2.11) converges to $f$ in the $L^{2}$ norm.

In the theory of spherical harmonics, a function or measure on $\mathbb{S}^{n-1}$ which is $\mathrm{SO}(n-1)$ invariant is often called zonal. The subspace of zonal functions in $\mathcal{H}_{k}^{n}$ is 1-dimensional for every $k \in \mathbb{N}$ and spanned by the function $u \mapsto P_{k}^{n}(u \cdot \bar{e})$, where $P_{k}^{n} \in C([-1,1])$ denotes the Legendre polynomial of dimension $n$ and degree $k$. Since the spaces $\mathcal{H}_{k}^{n}$ are orthogonal, it is not difficult to show that any zonal function $f \in L^{2}\left(\mathbb{S}^{n-1}\right)$ admits a series expansion of the form

$$
f \sim \sum_{k=0}^{\infty} \frac{N(n, k)}{\omega_{n}} a_{k}^{n}[f] P_{k}^{n}(\cdot \cdot \bar{e}),
$$

where $\omega_{n}$ denotes the surface area of the $n$-dimensional Euclidean unit ball and

$$
a_{k}^{n}[f]=\omega_{n-1} \int_{-1}^{1} f(t) P_{k}^{n}(t)\left(1-t^{2}\right)^{\frac{n-3}{2}} d t .
$$

Here, we have used again the cylindrical coordinates (2.2) to identify the zonal function $f$ with a function on $[-1,1]$.

Now we turn to (formal) Fourier expansions of measures and, more general, distributions on $\mathbb{S}^{n-1}$. To this end, first recall that a distribution on $\mathbb{S}^{n-1}$ is a continuous linear functional on $C^{\infty}\left(\mathbb{S}^{n-1}\right)$. Since $\mathbb{S}^{n-1}$ is compact, every distribution $\nu$ on $\mathbb{S}^{n-1}$ is of finite order, that is, there exist $k \in \mathbb{N}$ and $C>0$ such that

$$
|\nu(f)| \leq C\|f\|_{C^{k}}
$$

for every $f \in C^{\infty}\left(\mathbb{S}^{n-1}\right)$. The order of $\nu$ is the smallest $k$ such that (2.14) holds.

Examples of distributions on a general smooth manifold $M$ are smooth densities on $M$. Therefore, distributions are often also called generalized densities and $C^{-\infty}(|\Lambda|(M))$ is used to denote the space of distributions on $M$. However, the choice of a Riemannian metric on $M$ induces an isomorphism between the space of generalized densities and the space of generalized functions on $M$, usually denoted by $C^{-\infty}(M)$ (cf. [29]). Throughout this article, when $M=\mathbb{S}^{n-1}$,we always make use of this identification and, thus, write $C^{-\infty}\left(\mathbb{S}^{n-1}\right)$ for the space of distributions on $\mathbb{S}^{n-1}$ equipped with the topology of weak convergence. The canonical bilinear pairing on $C^{\infty}\left(\mathbb{S}^{n-1}\right) \times C^{-\infty}\left(\mathbb{S}^{n-1}\right)$ will be denoted by $\langle\cdot, \cdot\rangle$.

Since every (signed) Borel measure $\mu$ on $\mathbb{S}^{n-1}$ defines a distribution $\nu_{\mu}$ by

$$
\left\langle f, \nu_{\mu}\right\rangle=\int_{\mathbb{S}^{n-1}} f(u) d \mu(u), \quad f \in C^{\infty}\left(\mathbb{S}^{n-1}\right),
$$


we will use the continuous linear injection $\mu \mapsto \nu_{\mu}$, to identify $\mathcal{M}\left(\mathbb{S}^{n-1}\right)$ with a subspace of $C^{-\infty}\left(\mathbb{S}^{n-1}\right)$. Clearly, this subspace consists precisely of the distributions of order 0 . In the same way, the spaces $C^{\infty}\left(\mathbb{S}^{n-1}\right), C\left(\mathbb{S}^{n-1}\right)$, and $L^{2}\left(\mathbb{S}^{n-1}\right)$ can be identified with (dense) subspaces of $C^{-\infty}\left(\mathbb{S}^{n-1}\right)$ and we have

$$
C^{\infty}\left(\mathbb{S}^{n-1}\right) \subseteq C\left(\mathbb{S}^{n-1}\right) \subseteq L^{2}\left(\mathbb{S}^{n-1}\right) \subseteq \mathcal{M}\left(\mathbb{S}^{n-1}\right) \subseteq C^{-\infty}\left(\mathbb{S}^{n-1}\right)
$$

The natural action of $\mathrm{SO}(n)$ on $C^{-\infty}\left(\mathbb{S}^{n-1}\right)$ is defined as follows: For $\vartheta \in \mathrm{SO}(n)$ and $\nu \in C^{-\infty}\left(\mathbb{S}^{n-1}\right)$, we set

$$
\langle f, \vartheta \nu\rangle=\left\langle\vartheta^{-1} f, \nu\right\rangle, \quad f \in C^{\infty}\left(\mathbb{S}^{n-1}\right) .
$$

Note that if $\nu$ is a measure on $\mathbb{S}^{n-1}$, then $\vartheta \nu$ is just the image measure of $\nu$ under the rotation $\vartheta$ and that $(2.16)$ is also consistent with the left regular representation of $\mathrm{SO}(n)$ on the spaces $C^{\infty}\left(\mathbb{S}^{n-1}\right), C\left(\mathbb{S}^{n-1}\right)$, and $L^{2}\left(\mathbb{S}^{n-1}\right)$. We also remark that the action $\mathrm{SO}(n) \times C^{-\infty}\left(\mathbb{S}^{n-1}\right) \rightarrow C^{-\infty}\left(\mathbb{S}^{n-1}\right)$ is not continuous if $C^{-\infty}\left(\mathbb{S}^{n-1}\right)$ is endowed with the weak topology, but it is continuous in the strong topology, that is, the topology of uniform convergence on bounded sets.

In order to extend the orthogonal projection $\pi_{k}: L^{2}\left(\mathbb{S}^{n-1}\right) \rightarrow \mathcal{H}_{k}^{n}$ to $C^{-\infty}\left(\mathbb{S}^{n-1}\right)$, note that $\pi_{k}$ is self-adjoint. In particular, $\left\langle f, \pi_{k} g\right\rangle=\left\langle\pi_{k} f, g\right\rangle$ for all $f \in C^{\infty}\left(\mathbb{S}^{n-1}\right)$ and $g \in L^{2}\left(\mathbb{S}^{n-1}\right)$. In view of $(2.15)$, it is therefore consistent to define $\pi_{k} \nu$ for $\nu \in C^{-\infty}\left(\mathbb{S}^{n-1}\right)$ as the distribution given by

$$
\left\langle f, \pi_{k} \nu\right\rangle=\left\langle\pi_{k} f, \nu\right\rangle, \quad f \in C^{\infty}\left(\mathbb{S}^{n-1}\right) .
$$

From this, it follows (cf. [49, p. 38]) that indeed $\pi_{k} \nu \in \mathcal{H}_{k}^{n}$ for every $k \in \mathbb{N}$.

Next let us discuss the convolution of functions and measures on $\mathbb{S}^{n-1}$. Recall that the convolution $\sigma * \mu$ of signed measures $\sigma, \mu$ on $\mathrm{SO}(n)$ can be defined by

$$
\int_{\mathrm{SO}(n)} f(\vartheta) d(\sigma * \mu)(\vartheta)=\int_{\mathrm{SO}(n)} \int_{\mathrm{SO}(n)} f(\eta \theta) d \sigma(\eta) d \mu(\theta), \quad f \in C(\mathrm{SO}(n)) .
$$

In other words, $\sigma * \mu$ is the pushforward of the product measures $\sigma \otimes \mu$ by the group multiplication $m: \mathrm{SO}(n) \times \mathrm{SO}(n) \rightarrow \mathrm{SO}(n)$, that is, $\sigma * \mu=m_{*}(\sigma \otimes \mu)$. Since $\mathrm{SO}(n)$ is compact, this definition can be readily extended to distributions by replacing the product measure with the tensor product of distributions (see, e.g., [34, p. 128]).

The identification of $\mathbb{S}^{n-1}$ with the homogeneous space $\mathrm{SO}(n) / \mathrm{SO}(n-1)$ can now be used to identify $C^{-\infty}\left(\mathbb{S}^{n-1}\right)$ with right $\mathrm{SO}(n-1)$ invariant distributions on $\mathrm{SO}(n)$. Using this correspondence, the convolution of distributions on $\mathrm{SO}(n)$ induces a convolution product on $C^{-\infty}\left(\mathbb{S}^{n-1}\right)$ as follows: Let $\pi: \mathrm{SO}(n) \rightarrow \mathbb{S}^{n-1}$, $\pi(\eta)=\eta \bar{e}$, be the canonical projection. Then the convolution of distributions $\delta, \nu \in C^{-\infty}\left(\mathbb{S}^{n-1}\right)$ is defined by

$$
\delta * \nu=\pi_{*} m_{*}\left(\pi^{*} \delta \otimes \pi^{*} \nu\right)
$$

where $\pi_{*}$ and $\pi^{*}$ denote the pushforward and pullback by $\pi$, respectively. 
The convolution product defined in this way has the following well-known continuity property (see, e.g., [34, Chapter 6]).

Lemma 2.5 If $\nu_{j} \in C^{-\infty}\left(\mathbb{S}^{n-1}\right), j \in \mathbb{N}$, converge weakly to $\nu \in C^{-\infty}\left(\mathbb{S}^{n-1}\right)$, then $\lim _{j \rightarrow \infty} \delta * \nu_{j}=\delta * \nu$ weakly for every $\delta \in C^{-\infty}\left(\mathbb{S}^{n-1}\right)$.

Zonal measures play an essential role for spherical convolution. For later use we state here explicitly the expressions for the convolution of a function $h \in C\left(\mathbb{S}^{n-1}\right)$ and a measure $\sigma \in \mathcal{M}\left(\mathbb{S}^{n-1}\right)$ with a zonal measure $\mu \in \mathcal{M}\left(\mathbb{S}^{n-1}\right)$ and a zonal function $f \in C\left(\mathbb{S}^{n-1}\right)$, respectively:

$$
(h * \mu)(\bar{\eta})=\int_{\mathbb{S}^{n-1}} h(\eta u) d \mu(u) \quad \text { and } \quad(\sigma * f)(\bar{\eta})=\int_{\mathbb{S}^{n-1}} f\left(\eta^{-1} u\right) d \sigma(u),
$$

where for $\eta \in \mathrm{SO}(n)$, we write $\pi(\eta)=\bar{\eta} \in \mathbb{S}^{n-1}$.

From (2.17) one can deduce several properties of the convolution with zonal measures, for example, that the convolution of zonal functions and measures is Abelian and that for every $\vartheta \in \mathrm{SO}(n)$, we have $(\vartheta \sigma) * \mu=\vartheta(\sigma * \mu)$. Moreover, from the identification of the zonal measure $\mu$ on $\mathbb{S}^{n-1}$ with a measure on $[-1,1]$ and the well-known Funk-Hecke Theorem, it follows (cf. [59]) that the Fourier expansion of $\sigma * \mu$ is given by

$$
\sigma * \mu \sim \sum_{k=0}^{\infty} a_{k}^{n}[\mu] \pi_{k} \sigma,
$$

where the numbers $a_{k}^{n}[\mu]$ are defined by

$$
a_{k}^{n}[\mu]=\omega_{n-1} \int_{-1}^{1} P_{k}^{n}(t)\left(1-t^{2}\right)^{\frac{n-3}{2}} d \mu(t) .
$$

Like the convolution of functions on $\mathbb{R}^{n}$, spherical convolution can be used to approximate a given function or distribution on $\mathbb{S}^{n-1}$ by smooth functions. To this end, let $B_{j}(\bar{e}), j \in \mathbb{N}$, denote the open geodesic ball of radius $\frac{1}{j}$ centered at $\bar{e} \in \mathbb{S}^{n-1}$. A sequence of non-negative zonal functions $h_{j} \in C^{\infty}\left(\mathbb{S}^{n-1}\right), j \in \mathbb{N}$, is called a spherical approximate identity if for each $j$,

$$
\int_{\mathbb{S}^{n-1}} h_{j}(u) d u=1 \quad \text { and } \quad \operatorname{supp} h_{j} \subseteq B_{j}(\bar{e}) .
$$

For a proof of the following auxiliary result, we refer to [27] or [53, Chapter 6].

Lemma 2.6 If $h_{j} \in C^{\infty}\left(\mathbb{S}^{n-1}\right), j \in \mathbb{N}$, is a spherical approximate identity, then

(i) $\lim _{j \rightarrow \infty} g * h_{j}=g$ uniformly for every $g \in C\left(\mathbb{S}^{n-1}\right)$;

(ii) $\lim _{j \rightarrow \infty} \nu * h_{j}=\nu$ weakly for every $\nu \in C^{-\infty}\left(\mathbb{S}^{n-1}\right)$. 
In the final part of this section, we turn to the Christoffel problem and its solution by Berg [12]. First recall that spherical harmonics are eigenfunctions of the Laplacian $\Delta_{\mathbb{S}}$, more precisely, for $Y_{k} \in \mathcal{H}_{k}^{n}$,

$$
\Delta_{\mathbb{S}} Y_{k}=-k(k+n-2) Y_{k}
$$

Like the orthogonal projection $\pi_{k}$, the Laplacian $\Delta_{\mathbb{S}}$ is self-adjoint. Thus, it is consistent to define $\Delta_{\mathbb{S}} \nu$ for $\nu \in C^{-\infty}\left(\mathbb{S}^{n-1}\right)$ as the distribution given by

$$
\left\langle f, \Delta_{\mathbb{S}} \nu\right\rangle=\left\langle\Delta_{\mathbb{S}} f, \nu\right\rangle, \quad f \in C^{\infty}\left(\mathbb{S}^{n-1}\right) .
$$

In particular, by (2.15), $\Delta_{\mathbb{S}}$ can now be applied to support functions of not necessarily smooth convex bodies. This is important for us, since the first-order area measure $S_{1}(K, \cdot)$ of $K \in \mathcal{K}^{n}$ and its support function $h_{K}$ are related by a linear differential operator $\square_{n}$ in the following way:

$$
S_{1}(K, \cdot)=h_{K}+\frac{1}{n-1} \Delta_{\mathbb{S}} h_{K}=: \square_{n} h_{K}
$$

From (2.20) and the definition of $\square_{n}$, it follows that for $f \in C^{\infty}\left(\mathbb{S}^{n-1}\right)$, the spherical harmonic expansion of $\square_{n} f$ is given by

$$
\square_{n} f \sim \sum_{k=0}^{\infty} \frac{(1-k)(k+n-1)}{n-1} \pi_{k} f .
$$

Hence, the kernel of $\square_{n}: C^{\infty}\left(\mathbb{S}^{n-1}\right) \rightarrow C^{\infty}\left(\mathbb{S}^{n-1}\right)$ is given by $\mathcal{H}_{1}^{n}$, that is, it consists precisely of the restrictions of linear functions on $\mathbb{R}^{n}$ to $\mathbb{S}^{n-1}$. In the following let

$$
C_{\mathrm{o}}^{\infty}\left(\mathbb{S}^{n-1}\right)=\left\{f \in C^{\infty}\left(\mathbb{S}^{n-1}\right): \pi_{1} f=0\right\}
$$

and define $C_{\mathrm{o}}^{-\infty}\left(\mathbb{S}^{n-1}\right)$ in the same way. Then $\square_{n}: C_{\mathrm{o}}^{\infty}\left(\mathbb{S}^{n-1}\right) \rightarrow C_{\mathrm{o}}^{\infty}\left(\mathbb{S}^{n-1}\right)$ is an $\mathrm{SO}(n)$ equivariant isomorphism of topological vector spaces.

The classical Christoffel problem consists in finding necessary and sufficient conditions for a Borel measure on $\mathbb{S}^{n-1}$ to be the first-order area measure of a convex body. A solution was obtained by Berg [12] by providing an explicit inversion formula for the operator $\square_{n}$. He proved that for every $n \geq 2$ there exists a uniquely determined $C^{\infty}$ function $g_{n}$ on $(-1,1)$ such that the associated zonal function $\breve{g}_{n}(u)=g_{n}(u \cdot \bar{e})$ is in $L^{1}\left(\mathbb{S}^{n-1}\right)$ and

$$
a_{1}^{n}\left[g_{n}\right]=0, \quad a_{k}^{n}\left[g_{n}\right]=\frac{n-1}{(1-k)(k+n-1)}, \quad k \neq 1 .
$$

It follows from (2.22), (2.18), and (2.23) that for every $f \in C_{\mathrm{o}}^{\infty}\left(\mathbb{S}^{n-1}\right)$,

$$
f=\left(\square_{n} f\right) * \breve{g}_{n}
$$


In the final section, we need a generalization of (2.24) that can be deduced from [25. Theorem 4.3] and was independently proved in [11]: For every $j \in\{2, \ldots, n\}$, the integral transform

$$
\mathrm{T}_{g_{j}}: C_{\mathrm{o}}^{\infty}\left(\mathbb{S}^{n-1}\right) \rightarrow C_{\mathrm{o}}^{\infty}\left(\mathbb{S}^{n-1}\right), \quad f \mapsto f * \breve{g}_{j},
$$

is an isomorphism. We denote by $\square_{j}: C_{\mathrm{o}}^{\infty}\left(\mathbb{S}^{n-1}\right) \rightarrow C_{\mathrm{o}}^{\infty}\left(\mathbb{S}^{n-1}\right)$ the inverse of $\mathrm{T}_{g_{j}}$.

\section{Smooth and Generalized Valuations}

We now turn to the background material on translation invariant scalar and convex body valued valuations. In particular, we recall the definitions of smooth and generalized (spherical) valuations as well as the Alesker-Poincaré duality map.

If $G$ is a group of affine transformations on $\mathbb{R}^{n}$, a valuation $\phi$ is called $G$-invariant if $\phi(g K)=\phi(K)$ for all $K \in \mathcal{K}^{n}$ and every $g \in G$. Let Val denote the vector space of continuous translation invariant scalar valued valuations on $\mathbb{R}^{n}$. It was first proved by McMullen [47 that

$$
\mathrm{Val}=\bigoplus_{0 \leq i \leq n} \mathrm{Val}_{i}
$$

where $\mathbf{V a l}_{i} \subseteq \mathbf{V a l}$ denotes the subspace of valuations (homogeneous) of degree $i$.

Recall that a map $\Phi: \mathcal{K}^{n} \rightarrow \mathcal{K}^{n}$ is called a Minkowski valuation if

$$
\Phi K+\Phi L=\Phi(K \cup L)+\Phi(K \cap L)
$$

whenever $K \cup L$ is convex and addition on $\mathcal{K}^{n}$ is Minkowski addition. We denote by MVal the set of continuous translation invariant Minkowski valuations, and we write $\mathbf{M V a l}_{i}, 0 \leq i \leq n$, for its subset of Minkowski valuations of degree $i$.

More general than Minkowski valuations, we also consider valuations with values in $C\left(\mathbb{S}^{n-1}\right)$, that is, maps $F: \mathcal{K}^{n} \rightarrow C\left(\mathbb{S}^{n-1}\right), K \mapsto F_{K}$, such that

$$
F_{K}+F_{L}=F_{K \cup L}+F_{K \cap L}
$$

whenever $K \cup L$ is convex. Let $\mathbf{C V a l}$ denote the vector space of all such valuations which are continuous and translation invariant and, as before, let $\mathbf{C V a l}_{i}, 0 \leq i \leq n$, denote its subspace of valuations of degree $i$.

Note that any Minkowski valuation $\Phi \in$ MVal induces a valuation $F^{\Phi} \in \mathbf{C V a l}$ by $F_{K}^{\Phi}=h_{\Phi K}$, and that $\Phi$ is $\mathrm{SO}(n)$ equivariant if and only if $F^{\Phi}$ is $\mathrm{SO}(n)$ equivariant. Using the map $\Phi \mapsto F^{\Phi}$, the set $\mathbf{M V a l}$ can be identified with an infinite dimensional convex cone in CVal.

Clearly, a valuation $F \in \mathbf{C V a l}$ is uniquely determined by the family of valuations $\varphi_{u} \in$ Val, $u \in \mathbb{S}^{n-1}$, defined by $\varphi_{u}(K)=F_{K}(u)$ for $K \in \mathcal{K}^{n}$. If in addition $F$ is $\mathrm{SO}(n)$ equivariant, then for $\eta \in \mathrm{SO}(n)$ and $\bar{\eta}=\eta \bar{e} \in \mathbb{S}^{n-1}$,

$$
\varphi_{\bar{\eta}}(K)=F_{K}(\eta \bar{e})=\left(\eta^{-1} F_{K}\right)(\bar{e})=F_{\eta^{-1} K}(\bar{e})=\varphi_{\bar{e}}\left(\eta^{-1} K\right) .
$$


Hence, an $\mathrm{SO}(n)$ equivariant $F \in \mathbf{C V a l}$ is uniquely determined by a single $\mathrm{SO}(n-1)$ invariant valuation $\varphi_{\bar{e}} \in \mathrm{Val}$. In fact, there is a one-to-one correspondence between the subspace of $\mathrm{SO}(n-1)$ invariant valuations in Val and the subspace of $\mathrm{SO}(n)$ equivariant valuations in CVal. This observation leads to the following:

Definition Suppose that $F \in \mathbf{C V a l}$ is $\mathrm{SO}(n)$ equivariant. The $\mathrm{SO}(n-1)$ invariant valuation $\varphi \in \mathrm{Val}$, defined by

$$
\varphi(K)=F_{K}(\bar{e}), \quad K \in \mathcal{K}^{n},
$$

is called the associated real valued valuation of $F \in \mathbf{C V a l}$.

The following collection of examples and results on homogeneous valuations will be useful for later reference.

\section{Examples 3.1}

(a) It is a trivial fact that $\mathbf{V a l}_{0}$ is one-dimensional and spanned by the Euler characteristic $V_{0}$. (Recall that $V_{0}(K)=1$ for every $K \in \mathcal{K}^{n}$.) Using this observation, it follows that $\Phi_{0} \in \mathbf{M V a l}_{0}$ if and only if there exists an $L_{0} \in \mathcal{K}^{n}$ such that $\Phi_{0} K=L_{0}$ for every $K \in \mathcal{K}^{n}$. If $\Phi_{0}$ is also $\mathrm{SO}(n)$ equivariant, then $L_{0}=c_{0} B$, where $B$ denotes the Euclidean unit ball in $\mathbb{R}^{n}$ and $c_{0} \geq 0$.

Hadwiger [33, p. 79] proved that also $\mathbf{V a l}_{n}$ is one-dimensional and spanned by the ordinary volume $V_{n}$. From this one can easily deduce that $\Phi_{n} \in \mathbf{M V a l}_{n}$ if and only if there exists an $L_{n} \in \mathcal{K}^{n}$ such that $\Phi_{n} K=L_{n} V(K)$ for every $K \in \mathcal{K}^{n}$. If $\Phi_{n}$ is also $\mathrm{SO}(n)$ equivariant, then $L_{n}=c_{n} B$ for some $c_{n} \geq 0$.

(b) It was first proved by Spiegel [63] that if $\psi \in \mathbf{V a l}_{1}$, then

$$
\psi(K+L)=\psi(K)+\psi(L)
$$

for all $K, L \in \mathcal{K}^{n}$. Using this Minkowski additivity, a description of valuations in $\mathbf{V a l}_{1}$ was obtained by Goodey and Weil [23] and refined by Kiderlen [36]. In order to state their result, recall that any $f \in C^{\infty}\left(\mathbb{S}^{n-1}\right)$ can be written as a difference of two support functions $f=h_{K_{f}}-h_{r_{f} B}$, where $r_{f} \geq 0$ (see, e.g., [56, Lemma 1.7.8]). Now for $\psi \in \mathbf{V a l}_{1}$, let $\nu_{\psi} \in C_{\mathrm{o}}^{-\infty}\left(\mathbb{S}^{n-1}\right)$ be given by

$$
\left\langle f, \nu_{\psi}\right\rangle=\psi\left(K_{f}\right)-\psi\left(r_{f} B\right), \quad f \in C^{\infty}\left(\mathbb{S}^{n-1}\right),
$$

which is well defined by the Minkowski additivity of $\psi$. Moreover, since $r_{f}$ depends continuously on $f$ in the $C^{2}$ norm, the distribution $\nu_{\psi} \in C_{\mathrm{o}}^{-\infty}\left(\mathbb{S}^{n-1}\right)$ is of order at most 2. This allows one to conclude that if $\psi \in \mathbf{V a l}_{1}$, then there exists a uniquely determined $\nu_{\psi} \in C_{\mathrm{o}}^{-\infty}\left(\mathbb{S}^{n-1}\right)$ of order at most 2 , which can be extended to the vector space spanned by support functions, such that

$$
\psi(K)=\left\langle h_{K}, \nu_{\psi}\right\rangle
$$

for every $K \in \mathcal{K}^{n}$. Also observe that $\psi \mapsto \nu_{\psi}$ is continuous as a map from $\mathrm{Val}_{1}$ to $C_{\mathrm{o}}^{-\infty}\left(\mathbb{S}^{n-1}\right)$. 
Using this description of valuations in $\mathrm{Val}_{1}$, Kiderlen [36 proved that if $\Phi_{1} \in \mathrm{MVal}_{1}$ is $\mathrm{SO}(n)$ equivariant, then there exists a uniquely determined zonal $\nu_{1} \in C_{\mathrm{o}}^{-\infty}\left(\mathbb{S}^{n-1}\right)$ of order at most 2 such that

$$
h_{\Phi_{1} K}=h_{K} * \nu_{1}
$$

for every $K \in \mathcal{K}^{n}$. From (3.3) and a straightforward generalization of (2.18), it follows that for every $\Phi_{1} \in \mathbf{M V a l}_{1}$, there exists a uniquely determined sequence of real numbers $a_{k}^{n}\left[\Phi_{1}\right], k \in \mathbb{N}$, such that $a_{1}^{n}\left[\Phi_{1}\right]=0$ and

$$
\pi_{k} h_{\Phi_{1} K}=a_{k}^{n}\left[\Phi_{1}\right] \pi_{k} h_{K}
$$

for every $K \in \mathcal{K}^{n}$ and $k \in \mathbb{N}$. In fact, relation (3.4) was already proved by Schneider [54] in 1974, where he also showed that for every $k \neq 1$,

$$
\left|a_{k}^{n}\left[\Phi_{1}\right]\right| \leq a_{0}^{n}\left[\Phi_{1}\right] .
$$

We note that a precise description of the cone of zonal distributions in $C_{\mathrm{o}}^{-\infty}\left(\mathbb{S}^{n-1}\right)$ which generate a Minkowski valuation by (3.3) is still open. However, Kiderlen [36] showed that this cone contains all non-negative zonal measures on $\mathbb{S}^{n-1}$. More precisely, if $\mu_{1} \in \mathcal{M}_{\mathrm{o}}\left(\mathbb{S}^{n-1}\right)$ is zonal and non-negative, then

$$
h_{\Psi_{1} K}=h_{K} * \mu_{1}, \quad K \in \mathcal{K}^{n},
$$

defines an $\mathrm{SO}(n)$ equivariant Minkowski valuation in $\mathbf{M V a l}_{1}$.

(c) A classification of continuous translation invariant scalar valued valuations of degree $n-1$ was obtained by McMullen [48]. It states that $\phi \in \mathrm{Val}_{n-1}$ if and only if there exists a uniquely determined $f \in C_{\mathrm{o}}\left(\mathbb{S}^{n-1}\right)$ such that

$$
\phi(K)=\int_{\mathbb{S}^{n-1}} f(u) d S_{n-1}(K, u)
$$

for every $K \in \mathcal{K}^{n}$.

Applying McMullen's result to associated real valued valuations, the first author [59] obtained the following description of $\mathrm{SO}(n)$ equivariant Minkowski valuations of degree $n-1$ : If $\Phi_{n-1} \in \mathbf{M V a l}_{n-1}$ is $\mathrm{SO}(n)$ equivariant, then there exists a uniquely determined zonal $f_{n-1} \in C_{\mathrm{o}}\left(\mathbb{S}^{n-1}\right)$ such that

$$
h_{\Phi_{n-1} K}=S_{n-1}(K, \cdot) * f_{n-1}
$$

for every $K \in \mathcal{K}^{n}$. As in the case of Minkowski valuations of degree 1 , a precise description of the cone of zonal functions in $C_{\mathrm{o}}\left(\mathbb{S}^{n-1}\right)$ which generate a Minkowski valuation by (3.6) is still open (see [59] for more information). 
(d) Several important Minkowski valuations arise from data about sections or projections of convex bodies and are therefore objects of intensive research in geometric tomography (see, e.g., [21, 30, 36, 40, 41]). Of particular interest for us are the normalized mean section operators $\mathrm{M}_{j} \in \mathbf{M V a l}_{n+1-j}, 2 \leq j \leq n$, introduced by Goodey and Weil [24]. In [25. Theorem 4.4], they showed that for $K \in \mathcal{K}^{n}$,

$$
h_{\mathrm{M}_{j} K}=q_{n, j} S_{n+1-j}(K, \cdot) * \breve{g}_{j},
$$

where $g_{j}$ is the $j$ th Berg function and the constant $q_{n, j}$ is given by

$$
q_{n, j}=\frac{j-1}{2 \pi(n+1-j)} \frac{\kappa_{j-1} \kappa_{j-2} \kappa_{n-j}}{\kappa_{j-3} \kappa_{n-2}} .
$$

Here, $\kappa_{i}$ is the $i$-dimensional volume of the $i$-dimensional Euclidean unit ball.

A simple consequence of (3.1) is that the space Val becomes a Banach space when endowed with the norm

$$
\|\phi\|=\sup \{|\phi(K)|: K \subseteq B\} .
$$

The natural continuous action of the general linear group $\operatorname{GL}(n)$ on the Banach space $\mathbf{V a l}$ is for $A \in \mathrm{GL}(n)$ given by

$$
(A \phi)(K)=\phi\left(A^{-1} K\right), \quad \phi \in \mathbf{V a l}, K \in \mathcal{K}^{n} .
$$

The notion of smooth vectors of a continuous representation now gives rise to the notion of smooth valuations, first introduced by Alesker [5].

Definition $A$ valuation $\phi \in \mathbf{V a l}$ is called smooth if the map $\mathrm{GL}(n) \rightarrow$ Val, defined by $A \mapsto A \phi$, is infinitely differentiable.

Note that smooth valuations are precisely the smooth vectors of the natural representation of $\mathrm{GL}(n)$ on $\mathbf{V a l}$. We therefore write $\mathbf{V a l}^{\infty}$ for the Fréchet space of smooth translation invariant valuations endowed with the Gårding topology (see Section 2). We denote the subspace of smooth valuations of degree $i$ by $\mathbf{V a l}_{i}^{\infty}$. By the general properties of smooth vectors discussed in Section 2, the spaces $\mathbf{V a l}_{i}^{\infty}$ are dense $\mathrm{GL}(n)$ invariant subspaces of $\mathbf{V a l}_{i}$ and from (3.1) one can deduce that

$$
\mathrm{Val}^{\infty}=\bigoplus_{0 \leq i \leq n} \mathbf{V a l}_{i}^{\infty}
$$

Since in this article we are mainly concerned with $\mathrm{SO}(n)$ equivariant valuations, the following result will be useful.

Proposition 3.2 A valuation $\phi \in \mathrm{Val}$ is smooth if and only if the restricted map $\mathrm{SO}(n) \rightarrow$ Val, $\vartheta \mapsto \vartheta \phi$, is smooth. Moreover, the Gairding topologies on $\mathbf{V a l}^{\infty}$ induced by the natural representations of $\mathrm{GL}(n)$ and $\mathrm{SO}(n)$ coincide. 
The first statement of Proposition 3.2 follows for example from a recent result of Bernstein and Krötz [18, Corollary 3.10] and the fact that the natural representation of $\mathrm{GL}(n)$ on $\mathbf{V a l}$ is admissible and of finite length (see [4]). The second statement is then a simple consequence of the open mapping theorem.

The one-to-one correspondence between $\mathrm{SO}(n)$ equivariant valuations in CVal and $\mathrm{SO}(n-1)$ invariant valuations in Val now motivates the following.

Definition Let $F \in \mathbf{C V a l}$ be $\mathrm{SO}(n)$ equivariant and let $\varphi \in$ Val be the associated real valued valuation of $F$. We define the norm of $F$ by

$$
\|F\|=\sup \{|\varphi(K)|: K \subseteq B\} .
$$

Furthermore, we call $F$ smooth if $\varphi$ is smooth.

While it is easy to see that McMullen's decomposition result (3.1) implies that

$$
\mathbf{C V a l}=\bigoplus_{0 \leq i \leq n} \mathbf{C V a l}_{i}
$$

it was recently proved by Parapatits and the second author [51] that, in general, a Minkowski valuation $\Phi \in \mathbf{M V a l}$ need not be a sum of homogeneous Minkowski valuations $\Phi_{i} \in \mathbf{M V a l}_{i}$. However, from (3.9) one can still deduce a decomposition result for translation invariant Minkowski valuations (cf. [57]), which we state here under the additional assumption of $\mathrm{SO}(n)$ equivariance.

Lemma 3.3 If $\Phi \in \mathrm{MVal}$ is $\mathrm{SO}(n)$ equivariant, then there exist uniquely determined $c_{0}, c_{n} \geq 0$ and $\mathrm{SO}(n)$ equivariant valuations $F_{i} \in \mathbf{C V a l}_{i}, 1 \leq i \leq n-1$ such that

$$
h_{\Phi K}=c_{0}+\sum_{i=1}^{n-1} F_{i, K}+c_{n} V(K),
$$

for every $K \in \mathcal{K}^{n}$. Moreover, if $\Phi$ is smooth, then each $F_{i}$ is also smooth.

The vector space of $\mathrm{SO}(n)$ equivariant valuations in $\mathbf{C V a l}$ endowed with the norm (3.8) becomes a Banach space in which smooth valuations form a dense subspace. However, it is a priori not clear that an $\mathrm{SO}(n)$ equivariant Minkowski valuation in MVal can be approximated by smooth ones. We will prove this in Section 5 .

It is well known that for any $\phi \in \mathbf{V a l}$ and $K \in \mathcal{K}^{n}$, McMullen's decomposition (3.1) implies that the function $t \mapsto \phi(K+t B)$ is a polynomial of degree at most $n$. This, in turn, gives rise to a derivation operator $\Lambda:$ Val $\rightarrow$ Val, defined by

$$
(\Lambda \phi)(K)=\left.\frac{d}{d t}\right|_{t=0} \phi(K+t B) .
$$

Using (3.10) it is not difficult to show that if $\phi \in \mathbf{V a l}_{i}$, then $\Lambda \phi \in \mathbf{V a l}_{i-1}$, that $\Lambda$ is continuous, $\mathrm{SO}(n)$ equivariant, and that $\Lambda$ maps smooth valuations to smooth ones. 
The advantage of working with smooth translation invariant valuations instead of merely continuous ones is that the space $\mathbf{V a l}^{\infty}$ admits more algebraic structure. For example, the following Hard Lefschetz type theorem for the operator $\Lambda$ was proved by Alesker [5] for even and by Bernig and Bröcker [14] for general valuations.

Theorem $3.4\left([\mathbf{5},[14])\right.$ Suppose that $\frac{n}{2}<i \leq n$. Then $\Lambda^{2 i-n}: \mathbf{V a l}_{i}^{\infty} \rightarrow \mathbf{V a l}_{n-i}^{\infty}$ is an isomorphism.

Recently, Parapatits and the first author [50] proved that for any $\Phi \in \mathbf{M V a l}$, there exist $\Phi^{(j)} \in \mathbf{M V a l}$, where $0 \leq j \leq n$, such that

$$
\Phi(K+t B)=\sum_{j=0}^{n} t^{n-j} \Phi^{(j)}(K)
$$

for every $K \in \mathcal{K}^{n}$ and $t \geq 0$. This Steiner type formula shows that the operator $\Lambda$ from (3.10) has a natural analogue for Minkowski valuations $\Lambda:$ MVal $\rightarrow$ MVal.

Definition For $\Phi \in$ MVal, define $\Lambda \Phi \in$ MVal by

$$
h_{(\Lambda \Phi)(K)}(u)=\left.\frac{d}{d t}\right|_{t=0} h_{\Phi(K+t B)}(u), \quad u \in \mathbb{S}^{n-1} .
$$

Note that if $\Phi \in \mathbf{M V a l}_{i}$ is $\mathrm{SO}(n)$ equivariant, then so is $\Lambda \Phi \in \mathbf{M V a l} \mathbf{M}_{i-1}$. Moreover, if $\varphi \in \mathbf{V a l}_{i}$ is the associated real valued valuation of $\Phi$, then $\Lambda \varphi \in \mathbf{V a l}_{i-1}$ is associated with $\Lambda \Phi$. In particular, if $\Phi$ is smooth, then so is $\Lambda \Phi$.

Another important structural property of smooth valuations is the existence of a continuous bilinear product, discovered by Alesker [6],

$$
\cdot \mathbf{V a l}^{\infty} \times \mathrm{Val}^{\infty} \rightarrow \mathrm{Val}^{\infty}, \quad(\phi, \psi) \mapsto \phi \cdot \psi .
$$

Endowed with the Alesker product, $\mathbf{V a l}^{\infty}$ becomes an associative and commutative algebra with unit given by the Euler characteristic which is graded by the degree of homogeneity, that is,

$$
\mathrm{Val}_{i}^{\infty} \cdot \mathrm{Val}_{j}^{\infty} \subseteq \mathrm{Val}_{i+j}^{\infty} .
$$

Recall that $\mathbf{V a l}_{n}$ is 1-dimensional and spanned by $V_{n}$. If $V_{n}^{*} \in \mathbf{V a l}_{n}^{*}$ is the unique element such that $\left\langle V_{n}, V_{n}^{*}\right\rangle=1$, then it follows from (3.11) that for every $0 \leq i \leq n$,

$$
\langle\cdot, \cdot\rangle: \mathrm{Val}_{i}^{\infty} \times \mathrm{Val}_{n-i}^{\infty} \rightarrow \mathbb{R}, \quad(\phi, \psi) \mapsto\left\langle\phi \cdot \psi, V_{n}^{*}\right\rangle,
$$

defines a continuous bilinear pairing between smooth valuations of complementary degree. Moreover, Alesker [6] proved that this pairing is non-degenerate.

Definition The space of translation invariant generalized valuations of degree $i \in\{0, \ldots, n\}$ is defined as the topological dual

$$
\mathbf{V a l}_{i}^{-\infty}=\left(\mathbf{V a l}_{n-i}^{\infty}\right)^{*}
$$

endowed with the weak topology. 
Since the pairing (3.12) is non-degenerate, the Poincaré duality map, defined by

$$
\operatorname{pd}: \mathbf{V a l}_{i}^{\infty} \rightarrow \mathbf{V a l}_{i}^{-\infty}, \quad\langle\operatorname{pd} \phi, \psi\rangle=\langle\phi, \psi\rangle,
$$

is continuous, injective and has dense image with respect to the weak topology. Moreover, it follows from [8, Proposition 8.1.2] that pd admits a unique continuous extension to $\mathbf{V a l}_{i}$. Thus, we will use the Poincaré duality map to identify the spaces $\mathbf{V a l}_{i}^{\infty}$ and $\mathbf{V a l}_{i}$ with dense subspaces of $\mathbf{V a l}_{i}^{-\infty}$. Hence, we have the inclusions

$$
\mathrm{Val}_{i}^{\infty} \subseteq \mathrm{Val}_{i} \subseteq \mathrm{Val}_{i}^{-\infty}
$$

We also note that the natural action of $\mathrm{SO}(n)$ on $\mathbf{V a l}_{i}^{-\infty}$ is not continuous in the weak topology, but it is continuous when $\mathrm{Val}_{i}^{-\infty}$ is given the strong topology.

Next we recall a recent result of Alesker, Bernig and the first author [10] on the decomposition of the vector spaces of translation invariant (generalized) valuations into $\mathrm{SO}(n)$ irreducible subspaces.

Theorem 3.5 ([10]) For $0 \leq i \leq n$, the spaces $\mathbf{V a l}_{i}^{\infty}, \mathbf{V a l}_{i}$, and $\mathbf{V a l}_{i}^{-\infty}$ are multiplicity free under the action of $\mathrm{SO}(n)$. Moreover, the highest weights of the $\mathrm{SO}(n)$ irreducible subspaces in either of them are precisely given by the tuples $\left(\lambda_{1}, \ldots, \lambda_{\lfloor n / 2\rfloor}\right)$ satisfying (2.9) and the following additional conditions:

(i) $\lambda_{j}=0$ for $j>\min \{i, n-i\} ; \quad($ ii $)\left|\lambda_{j}\right| \neq 1$ for $1 \leq j \leq\left\lfloor\frac{n}{2}\right\rfloor ; \quad($ iii $)\left|\lambda_{2}\right| \leq 2$.

We now use the notion of spherical representations (see Section 2) to define spherical valuations.

Definition For $0 \leq i \leq n$, the subspaces $\mathbf{V a l}_{i}^{\mathrm{sph}}, \mathbf{V a l}_{i}^{\infty, \mathrm{sph}}$, and $\mathbf{V a l}_{i}^{-\infty, \mathrm{sph}}$ of translation invariant continuous, smooth, and generalized spherical valuations of degree $i$ are defined as the closures (w.r.t. the respective topologies) of the direct sum of all $\mathrm{SO}(n)$ irreducible subspaces in $\mathbf{V a l}_{i}, \mathbf{V a l}_{i}^{\infty}$, and $\mathbf{V a l}_{i}^{-\infty}$, respectively, which are spherical with respect to $\mathrm{SO}(n-1)$.

Note that, by Theorems 3.5 and 2.3 (see also Example 2.4), $\mathbf{V a l}_{i}^{-\infty, \mathrm{sph}}$ is the annihilator of the closure of the direct sum of all $\mathrm{SO}(n)$ irreducible subspaces in $\mathbf{V a l}_{n-i}^{\infty}$ with highest weights not of the form $(k, 0, \ldots, 0), k \in \mathbb{N}$. As a consequence, every $\mathrm{SO}(n-1)$ invariant (generalized) valuation in $\mathbf{V a l}_{i}, \mathbf{V a l}_{i}^{\infty}$, or $\mathbf{V a l}_{i}^{-\infty}$ is spherical.

\section{Examples 3.6}

(a) It follows from Theorem 3.5 or Example 3.1 (b) that

$$
\mathrm{Val}_{1}=\mathrm{Val}_{1}^{\mathrm{sph}}, \quad \mathrm{Val}_{1}^{\infty}=\mathrm{Val}_{1}^{\infty, \mathrm{sph}}, \quad \mathrm{Val}_{1}^{-\infty}=\mathrm{Val}_{1}^{-\infty, \mathrm{sph}} .
$$

(b) It follows from Theorem 3.5 or Example 3.1 (c) that

$$
\mathrm{Val}_{n-1}=\mathrm{Val}_{n-1}^{\mathrm{sph}}, \quad \mathrm{Val}_{n-1}^{\infty}=\mathrm{Val}_{n-1}^{\infty, \mathrm{sph}}, \quad \mathrm{Val}_{n-1}^{-\infty}=\mathrm{Val}_{n-1}^{-\infty, \mathrm{sph}}
$$




\section{Auxiliary Results about Smooth Valuations}

In this section we begin with the proof of Theorem 3 (a). As a corollary, we obtain a version of Theorem 2 for smooth Minkowski valuations. We also determine an explicit expression for the pairing (3.12) when one of the valuations is spherical. This will be needed in the next section to complete the proof of Theorem 3 (a).

Theorem 4.1 For $1 \leq i \leq n-1$, the map $\mathrm{E}_{i}: C_{\mathrm{o}}^{\infty}\left(\mathbb{S}^{n-1}\right) \rightarrow \mathbf{V a l}_{i}^{\infty, \mathrm{sph}}$, defined by

$$
\left(\mathrm{E}_{i} f\right)(K)=\int_{\mathbb{S}^{n-1}} f(u) d S_{i}(K, u)
$$

is an $\mathrm{SO}(n)$ equivariant isomorphism of topological vector spaces.

Proof. Clearly, the maps $\mathrm{E}_{i}: C_{\mathrm{o}}\left(\mathbb{S}^{n-1}\right) \rightarrow \mathbf{V a l}_{i}$, given by (4.1), are linear and $\mathrm{SO}(n)$ equivariant for every $i \in\{1, \ldots, n-1\}$. Using the monotonicity of the mixed volumes $V(K[i] ; B[n-i])$ with $i$ copies of $K$ and $n-i$ copies of the Euclidean unit ball $B$, we obtain for $K \subseteq B$,

$$
\left|\mathrm{E}_{i} f(K)\right| \leq \int_{\mathbb{S}^{n-1}}|f(u)| d S_{i}(K, u) \leq n V(K[i] ; B[n-i])\|f\| \leq n \kappa_{n}\|f\|,
$$

which proves $\left\|\mathrm{E}_{i} f\right\| \leq n \kappa_{n}\|f\|$ and, hence, the continuity of $\mathrm{E}_{i}$. Thus, by Lemma2.2 and Proposition 3.2, the restrictions $\mathrm{E}_{i}: C_{\mathrm{o}}^{\infty}\left(\mathbb{S}^{n-1}\right) \rightarrow \mathbf{V a l}_{i}^{\infty}$ are well defined and continuous.

Since differences of area measures of order $i$ of convex bodies in $\mathcal{K}^{n}$ are dense in the set of all signed finite Borel measures on $\mathbb{S}^{n-1}$ with centroid at the origin (see, e.g., [56, p. 477])), the maps $\mathrm{E}_{i}$ are also injective. Consequently, by Schur's lemma and Example 2.4, $\mathrm{E}_{i}\left(\mathcal{H}_{k}^{n}\right), k \neq 1$, is an $\mathrm{SO}(n)$ irreducible subspace of $\mathbf{V a l}_{i}^{\infty}$ of highest weight $(k, 0, \ldots, 0)$. By the definition of the spaces $\mathbf{V a l}_{i}^{\infty, \text { sph }}$, it follows that $\mathbf{U}_{i}:=\mathrm{E}_{i}\left(C_{\mathrm{o}}^{\infty}\left(\mathbb{S}^{n-1}\right)\right)$ is a dense subspace of $\mathbf{V a l}_{i}^{\infty, \mathrm{sph}}$. By the open mapping theorem, it remains to show that $\mathbf{U}_{i}$ is closed.

First, let $i=n-1$. The result of McMullen [48] discussed in Example 3.1 (c) implies that the map $\mathrm{E}_{n-1}: C_{\mathrm{o}}\left(\mathbb{S}^{n-1}\right) \rightarrow \mathbf{V a l}_{i}^{\text {sph }}$ is a continuous bijection and hence, by the open mapping theorem, an isomorphism of Banach spaces. The assertion for $i=n-1$ is now an immediate consequence of Lemma 2.2 and Proposition 3.2 .

Next, recall that the area measures satisfy the Steiner type formula

$$
S_{i}(K+t B, \cdot)=\sum_{j=0}^{i} t^{i-j}\left(\begin{array}{l}
i \\
j
\end{array}\right) S_{j}(K, \cdot)
$$

for every $K \in \mathcal{K}^{n}$ and $t \geq 0$. Thus, for $f \in C_{\mathrm{o}}^{\infty}\left(\mathbb{S}^{n-1}\right)$ and $i \geq 2$, we have

$$
\left(\Lambda \mathrm{E}_{i} f\right)(K)=\left.\frac{d}{d t}\right|_{t=0} \int_{\mathbb{S}^{n-1}} f(u) d S_{i}(K+t B, u)=i\left(\mathrm{E}_{i-1} f\right)(K) .
$$


In particular, the restriction of the derivation operator $\Lambda$ to $\mathbf{U}_{i}$ is injective for $2 \leq i \leq n-1$ and $\Lambda\left(\mathbf{U}_{i}\right)=\mathbf{U}_{i-1}$. Consequently, the restriction of the linear $\mathrm{SO}(n)$ equivariant map $\Lambda$ to $\mathrm{cl} \mathbf{U}_{i}=\mathbf{V a l}_{i}^{\infty, \text { sph }}$ is injective as well. By Theorem 3.4, $\Lambda^{n-2}: \mathbf{V a l}_{n-1}^{\infty} \rightarrow \mathbf{V a l}_{1}^{\infty}$ is an $\mathrm{SO}(n)$ equivariant isomorphism. But $\mathbf{U}_{n-1}=\mathbf{V a l}_{n-1}^{\infty}$ by what we have proved above. Therefore,

$$
\mathbf{U}_{1}=\Lambda^{n-2}\left(\mathbf{U}_{n-1}\right)=\mathbf{V a l}_{1}^{\infty} .
$$

In particular, $\mathbf{U}_{1}=\Lambda^{i-1}\left(\mathbf{U}_{i}\right)$ is closed. Hence, $\Lambda^{i-1}\left(\mathbf{U}_{i}\right) \subseteq \Lambda^{i-1}\left(\operatorname{cl} \mathbf{U}_{i}\right) \subseteq \Lambda^{i-1}\left(\mathbf{U}_{i}\right)$ that is,

$$
\Lambda^{i-1}\left(\mathbf{U}_{i}\right)=\Lambda^{i-1}\left(\mathrm{cl} \mathbf{U}_{i}\right) .
$$

Since $\Lambda^{i-1}$ is injective on $\mathrm{cl} \mathbf{U}_{i}=\mathbf{V a l}_{i}^{\infty, \text { sph }}$, we conclude that $\mathbf{U}_{i}$ is closed and, therefore, $\mathbf{U}_{i}=\mathbf{V a l}_{i}^{\infty, \mathrm{sph}}$.

Using Lemma 3.3 and Theorem 4.1, we can now prove the following Hadwiger type result for smooth Minkowski valuations.

Corollary 4.2 If $\Phi: \mathcal{K}^{n} \rightarrow \mathcal{K}^{n}$ is a smooth Minkowski valuation which is translation invariant and $\mathrm{SO}(n)$ equivariant, then there exist uniquely determined $c_{0}, c_{n} \geq 0$ and $\mathrm{SO}(n-1)$ invariant $f_{i} \in C_{\mathrm{o}}^{\infty}\left(\mathbb{S}^{n-1}\right), 1 \leq i \leq n-1$, such that

$$
h_{\Phi K}=c_{0}+\sum_{i=1}^{n-1} S_{i}(K, \cdot) * f_{i}+c_{n} V_{n}(K)
$$

for every $K \in \mathcal{K}^{n}$.

Proof. By Lemma 3.3, it is sufficient to show that for every $\mathrm{SO}(n)$ equivariant smooth $F_{i} \in \mathbf{C V a l}_{i}, 1 \leq i \leq n-1$, there exists a uniquely determined $\mathrm{SO}(n-1)$ invariant $f_{i} \in C_{\mathrm{o}}^{\infty}\left(\mathbb{S}^{n-1}\right)$, such that for every $K \in \mathcal{K}^{n}$,

$$
F_{i, K}=S_{i}(K, \cdot) * f_{i} .
$$

To this end, let $\varphi_{i} \in \mathbf{V a l}_{i}^{\infty}$ denote the associated real valued valuation of $F_{i}$ and recall that, by definition, $\varphi_{i}$ is $\operatorname{SO}(n-1)$ invariant. It follows that $\varphi_{i} \in \mathbf{V a l}_{i}^{\infty, \mathrm{sph}}$. Thus, by Theorem 4.1, there exists a uniquely determined $f_{i} \in C_{\mathrm{o}}^{\infty}\left(\mathbb{S}^{n-1}\right)$ such that

$$
\varphi_{i}(K)=\int_{\mathbb{S}^{n-1}} f_{i}(u) d S_{i}(K, u) .
$$

Moreover, the $\mathrm{SO}(n-1)$ invariance of $\varphi_{i}$ implies that also $f_{i}$ is $\mathrm{SO}(n-1)$ invariant. Hence, by the definition of $\varphi_{i}$ and (2.17), we obtain

$$
F_{i, K}(\bar{\eta})=\varphi_{i}\left(\eta^{-1} K\right)=\int_{\mathbb{S}^{n-1}} f_{i}\left(\eta^{-1} u\right) d S_{i}(K, u)=\left(S_{i}(K, \cdot) * f_{i}\right)(\bar{\eta}),
$$

where for $\eta \in \mathrm{SO}(n)$, we set, as before, $\bar{\eta}=\eta \bar{e} \in \mathbb{S}^{n-1}$.

Corollary 4.2 under the additional assumption that the Minkowski valuation $\Phi$ is even was recently obtained by the authors [62] using a different approach. 
Finally, we require a generalization of a formula of Bernig and Hug [17] for the pairing (3.12) of spherical valuations. To this end, let $a: \mathbb{S}^{n-1} \rightarrow \mathbb{S}^{n-1}$ denote the antipodal map, given by, $a(u)=-u, u \in \mathbb{S}^{n-1}$, and recall from Example 3.1 (b) that any $\psi \in \mathbf{V a l}_{1}$ determines a unique $\nu_{\psi} \in C_{\mathrm{o}}^{-\infty}\left(\mathbb{S}^{n-1}\right)$, defined by (3.2).

Proposition 4.3 Let $1 \leq i \leq n-1$. For $\phi_{i} \in \operatorname{Val}_{i}$ and $f \in C_{\mathrm{o}}^{\infty}\left(\mathbb{S}^{n-1}\right)$, we have

$$
\left\langle\phi_{i}, \mathrm{E}_{n-i} f\right\rangle=\frac{(n-i) !}{(n-1) !}\left\langle f \circ a, \nu_{\Lambda^{i-1} \phi_{i}}\right\rangle .
$$

Proof. Since both pairings in (4.5) are jointly continuous and bilinear, we may assume that $f \in \mathcal{H}_{k}^{n}$ for some $k \in \mathbb{N}, k \neq 1$, and that $\phi_{i}$ belongs to an $\mathrm{SO}(n)$ irreducible subspace $\Gamma_{\lambda} \subseteq \mathbf{V a l}_{i}$ of highest weight $\lambda=\left(\lambda_{1}, \ldots, \lambda_{\lfloor n / 2\rfloor}\right)$. In particular, $\phi_{i}$ is smooth.

Next, note that $\Lambda^{i-1} \phi_{i} \in \mathbf{V a l}_{1}^{\infty}=\mathbf{V a l}_{1}^{\infty, \text { sph }}$ (cf. Examples 3.6). Therefore, by Theorem 4.1, there exists a smooth function $h \in C_{\mathrm{o}}^{\infty}\left(\mathbb{S}^{n-1}\right)$ (in fact, $h \in \mathcal{H}_{m}^{n}$ for some $m \in \mathbb{N}$ ) such that

$$
\left(\Lambda^{i-1} \phi_{i}\right)(K)=i ! \int_{\mathbb{S}^{n-1}} h(u) d S_{1}(K, u) .
$$

The normalizing coefficient $i$ ! is chosen for convenience as will become clear below.

Since the pairing (3.12) is biinvariant under the (simultaneous) action of $\mathrm{SO}(n)$ and the spaces $\mathcal{H}_{k}^{n}$ are self-dual as $\mathrm{SO}(n)$ modules, the restriction of the Poincaré duality map to $\Gamma_{\lambda}$ defines a linear $\mathrm{SO}(n)$ equivariant map from $\Gamma_{\lambda}$ to $\mathcal{H}_{k}^{n}$, that is,

$$
\left.\operatorname{pd}\right|_{\Gamma_{\lambda}} \in \operatorname{Hom}_{\mathrm{SO}(n)}\left(\Gamma_{\lambda}, \mathcal{H}_{k}^{n}\right) .
$$

Since both $\Gamma_{\lambda}$ and $\mathcal{H}_{k}^{n}$ are $\mathrm{SO}(n)$ irreducible, it follows from Schur's lemma that $\left.\mathrm{pd}\right|_{\Gamma_{\lambda}}$ and, thus, the left hand side of (4.5) can only be non-zero when $\Gamma_{\lambda}$ and $\mathcal{H}_{k}^{n}$ are isomorphic, that is, when $\left(\lambda_{1}, \ldots, \lambda_{\lfloor n / 2\rfloor}\right)=(k, 0, \ldots, 0)$. Similarly, the right hand side of (4.5) can only be non-zero if $\left(\lambda_{1}, \ldots, \lambda_{\lfloor n / 2\rfloor}\right)=(k, 0, \ldots, 0)$. We may therefore assume that $\phi_{i}$ is spherical. But if $\phi_{i} \in \mathbf{V a l}_{i}^{\infty, \text { sph }}$, then, by (4.6) and (4.2), we have

$$
\phi_{i}(K)=\int_{\mathbb{S}^{n-1}} h(u) d S_{i}(K, u)=\mathrm{E}_{i} h .
$$

In this case, it follows from [17, Proposition 4.11] that

$$
\left\langle\mathrm{E}_{i} h, \mathrm{E}_{n-i} f\right\rangle=\frac{(n-i) ! i !}{(n-1) !} \int_{\mathbb{S}^{n-1}} h(u) \square_{n} f(-u) d u .
$$

Finally, definition (3.2), (4.6), and (2.21) yield

$$
\left\langle\phi_{i}, \mathrm{E}_{n-i} f\right\rangle=\frac{(n-i) !}{(n-1) !}\left\langle f \circ a, \nu_{\Lambda^{i-1} \phi_{i}}\right\rangle .
$$




\section{Proof of the Main Results}

We are now in a position to complete the proofs of Theorems 2 and 3 , We also discuss a more precise version of Theorem 2 for homogeneous Minkowski valuations in dimensions $n \leq 4$. At the end of the section we include an approximation result for continuous Minkowski valuations by smooth ones.

We begin with the following slightly more precise version of Theorem 3 (a).

Theorem 5.1 For $1 \leq i \leq n-1$, the isomorphism $\mathrm{E}_{i}: C_{\mathrm{o}}^{\infty}\left(\mathbb{S}^{n-1}\right) \rightarrow \mathbf{V a l}_{i}^{\infty, \mathrm{sph}}$ admits a unique extension by continuity in the weak topologies to an isomorphism

$$
\widetilde{\mathrm{E}}_{i}: C_{\mathrm{o}}^{-\infty}\left(\mathbb{S}^{n-1}\right) \rightarrow \mathrm{Val}_{i}^{-\infty, \mathrm{sph}}
$$

Moreover, the diagram

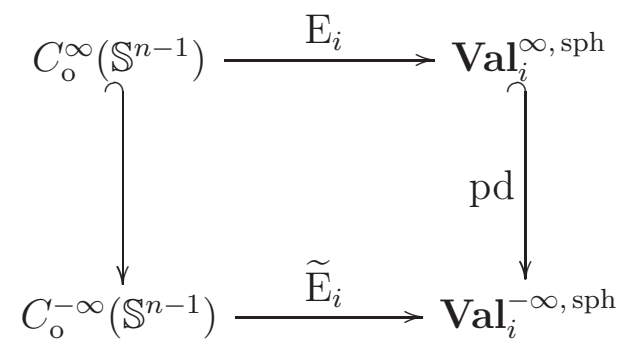

commutes and the vertical maps have dense image.

Proof. First recall that $\mathbf{V a l}_{i}^{-\infty, \text { sph }}$ is the annihilator of the subspace spanned by all $\mathrm{SO}(n)$ irreducible subspaces of $\mathbf{V a l}_{n-i}^{\infty}$ which are non-spherical. Hence, using Theorem 4.1, we can define a map $\widetilde{\mathrm{E}}_{i}: C_{\mathrm{o}}^{-\infty}\left(\mathbb{S}^{n-1}\right) \rightarrow \mathrm{Val}_{i}^{-\infty, \text { sph }}$ by

$$
\left\langle\widetilde{\mathrm{E}}_{i} \nu, \phi_{n-i}\right\rangle=\frac{i !}{(n-1) !}\left\langle\nu,\left(\square_{n} \circ a^{*} \circ \mathrm{E}_{1}^{-1} \circ \Lambda^{n-i}\right) \phi_{n-i}\right\rangle,
$$

where $\phi_{n-i} \in \mathbf{V a l}_{n-i}^{\infty}$ and $a^{*}$ denotes the pullback by the antipodal map. From (4.7), it follows that $\widetilde{\mathrm{E}}_{i}$ continuously extends $\mathrm{E}_{i}$.

Since the differential operator $\square_{n}: C_{\mathrm{o}}^{\infty}\left(\mathbb{S}^{n-1}\right) \rightarrow C_{\mathrm{o}}^{\infty}\left(\mathbb{S}^{n-1}\right)$ is an isomorphism, it follows from Theorem 4.1 that $\widetilde{\mathrm{E}}_{i} \nu=0$ implies $\nu=0$, that is, $\widetilde{\mathrm{E}}_{i}$ is injective. In order to prove that $\widetilde{\mathrm{E}}_{i}$ is surjective, let $\xi \in \mathbf{V a l}_{i}^{-\infty, \mathrm{sph}}$ be given and note that $\xi \circ \mathrm{E}_{n-i} \in C_{\mathrm{o}}^{-\infty}\left(\mathbb{S}^{n-1}\right)$. If we put

$$
\nu=\frac{(n-1) !}{(n-i) ! i !} \xi \circ \mathrm{E}_{n-i} \circ a^{*} \circ \square_{n}^{-1} \in C_{\mathrm{o}}^{-\infty}\left(\mathbb{S}^{n-1}\right),
$$

then, by (5.1),

$$
\left\langle\widetilde{\mathrm{E}}_{i} \nu, \mathrm{E}_{n-i} f\right\rangle=\left\langle\xi, \mathrm{E}_{n-i} f\right\rangle
$$

for all $f \in C_{\mathrm{o}}^{\infty}\left(\mathbb{S}^{n-1}\right)$, that is, $\widetilde{\mathrm{E}}_{i} \nu=\xi$. Clearly, the map $\widetilde{\mathrm{E}}_{i}^{-1}$ thus defined is continuous. 
In the next lemma, which is crucial for the proof of Theorem 3 (b), and in all that follows, the letter $C$ will denote a constant that can be different from one line to the next and that depends only on the dimension $n$.

Lemma 5.2 There exists a constant $C>0$ such that

$$
\|f\|_{C^{2}} \leq C\left\|\square_{n} f\right\|_{C^{0}} .
$$

for every $\mathrm{SO}(n-1)$ invariant $f \in C_{\mathrm{o}}^{2}\left(\mathbb{S}^{n-1}\right)$.

Proof. For arbitrary but fixed $q \in \mathbb{R}$, we consider the linear differential operator $\mathrm{D}_{q}: C^{2}\left(\mathbb{S}^{n-1}\right) \rightarrow C\left(\mathbb{S}^{n-1}\right)$, defined by

$$
\mathrm{D}_{q} f=\Delta_{\mathbb{S}} f+q f
$$

Note that $\mathrm{D}_{q}$ is $\mathrm{SO}(n)$ equivariant and that $\mathrm{D}_{n-1}=(n-1) \square_{n}$. Moreover, by (2.20), the operator $\mathrm{D}_{q}$ is injective for every $q \neq k(k+n-2), k \in \mathbb{N}$. If $q=k(k+n-2)$ for some $k \in \mathbb{N}$, then the kernel of $\mathrm{D}_{q}$ is given by $\mathcal{H}_{k}^{n}$.

First, we show that there exists a constant $C>0$ such that for $k=1,2$,

$$
\left|\nabla^{k} f\right|_{0}:=\max _{\mathbb{S}^{n-1}}\left|\nabla^{k} f\right| \leq C\left(\|f\|_{C^{0}}+\left\|\mathrm{D}_{q} f\right\|_{C^{0}}\right)
$$

for every $\mathrm{SO}(n-1)$ invariant $f \in C^{2}\left(\mathbb{S}^{n-1}\right)$ and, therefore, by (2.1),

$$
\|f\|_{C^{2}} \leq C\left(\|f\|_{C^{0}}+\left\|\mathrm{D}_{q} f\right\|_{C^{0}}\right) .
$$

Using the cylindrical coordinates (2.2) and expressions (2.7) and (2.8), we see that in order to prove (5.2), it suffices to prove that

$$
\left|\partial_{t} f\right|_{0}:=\sup _{(-1,1)}\left|\frac{\partial f}{\partial t}\right| \leq C\left(\|f\|_{C^{0}}+\left\|\mathrm{D}_{q} f\right\|_{C^{0}}\right)
$$

and

$$
\left|\left(1-t^{2}\right) \partial_{t t} f\right|_{0}:=\sup _{(-1,1)}\left|\left(1-t^{2}\right) \frac{\partial^{2} f}{\partial t^{2}}\right| \leq C\left(\|f\|_{C^{0}}+\left\|\mathrm{D}_{q} f\right\|_{C^{0}}\right)
$$

for every $\mathrm{SO}(n-1)$ invariant $f \in C^{2}\left(\mathbb{S}^{n-1}\right)$. But since $\left(1-t^{2}\right) \partial_{t t} f=\Delta_{\mathbb{S}} f+(n-1) t \partial_{t} f$ by (2.6), it follows that (5.5) is actually an immediate consequence of (5.4) and the definition of $\mathrm{D}_{q}$. Thus, in order to prove (5.2), we only have to show that (5.4) holds for every $\mathrm{SO}(n-1)$ invariant $f \in C^{2}\left(\mathbb{S}^{n-1}\right)$.

Let $f \in C^{2}\left(\mathbb{S}^{n-1}\right)$ now be an arbitrary but fixed $\mathrm{SO}(n-1)$ invariant function. Since $|\nabla f|$ and $\left|\nabla^{2} f\right|$ are bounded on $\mathbb{S}^{n-1}$, it follows from (2.7) and (2.8) that $\partial_{t} f$ is bounded on $(-1,1)$. Assume that $\left|\partial_{t} f\right|$ attains its maximum at $t_{0} \in(-1,1)$. Since, by (2.6),

$$
\Delta_{\mathbb{S}} f=\left(1-t^{2}\right) \partial_{t t} f-(n-1) t \partial_{t} f=\left(1-t^{2}\right)^{1-(n-1) / 2} \partial_{t}\left(\left(1-t^{2}\right)^{(n-1) / 2} \partial_{t} f\right),
$$


it follows from the definition of $\mathrm{D}_{q}$ that

$$
\begin{aligned}
\left(1-t_{0}^{2}\right)^{(n-1) / 2} \partial_{t} f\left(t_{0}\right) & =\int_{-1}^{t_{0}} \partial_{t}\left(\left(1-t^{2}\right)^{(n-1) / 2} \partial_{t} f(t)\right) d t \\
& =\int_{-1}^{t_{0}}\left(1-t^{2}\right)^{(n-1) / 2-1}\left(\mathrm{D}_{q} f(t)-q f(t)\right) d t
\end{aligned}
$$

and, hence,

$$
\left|\left(1-t_{0}^{2}\right)^{(n-1) / 2} \partial_{t} f\left(t_{0}\right)\right| \leq C\left(\|f\|_{C^{0}}+\left\|\mathrm{D}_{q} f\right\|_{C^{0}}\right) .
$$

This shows that we may assume that $\left|t_{0}\right| \geq \alpha$ for some fixed $\alpha>0$, otherwise (5.6) implies (5.4). But since $\partial_{t t} f\left(t_{0}\right)=0$, we conclude from (2.6) that

$$
-(n-1) t_{0} \partial_{t} f\left(t_{0}\right)=\mathrm{D}_{q} f\left(t_{0}\right)-q f\left(t_{0}\right)
$$

which also yields $\left|\partial_{t} f\left(t_{0}\right)\right| \leq C\left(\|f\|_{C^{0}}+\left\|\mathrm{D}_{q} f\right\|_{C^{0}}\right)$. Hence, we have shown that

$$
\left|\partial_{t} f\right|_{0} \leq \max \left\{C\left(\|f\|_{C^{0}}+\left\|\mathrm{D}_{q} f\right\|_{C^{0}}\right), \limsup _{t \rightarrow \pm 1}\left|\partial_{t} f(t)\right|\right\}
$$

and it remains to bound $\lim \sup _{t \rightarrow \pm 1}\left|\partial_{t} f(t)\right|$ in terms of $\|f\|_{C^{0}}$ and $\left\|\mathrm{D}_{q} f\right\|_{C^{0}}$. In order to do this, note that, by (2.6),$\partial_{t} f$ is a bounded solution on $(-1,1)$ of the differential equation

$$
y^{\prime}(t)-\frac{(n-1) t}{1-t^{2}} y(t)=\frac{\mathrm{D}_{q} f(t)-q f(t)}{1-t^{2}} .
$$

All solutions of this equation are given by

$$
y(t)=\left(1-t^{2}\right)^{-(n-1) / 2}\left(\int_{-1}^{t} \frac{\mathrm{D}_{q} f(s)-q f(s)}{\left(1-s^{2}\right)^{1-(n-1) / 2}} d s+c\right),
$$

where $c \in \mathbb{R}$. Since $\partial_{t} f$ is bounded, we must have

$$
\partial_{t} f(t)=\left(1-t^{2}\right)^{-(n-1) / 2} \int_{-1}^{t} \frac{\mathrm{D}_{q} f(s)-q f(s)}{\left(1-s^{2}\right)^{1-(n-1) / 2}} d s
$$

and

$$
\int_{-1}^{1} \frac{\mathrm{D}_{q} f(s)-q f(s)}{\left(1-s^{2}\right)^{1-(n-1) / 2}} d s=0
$$

Consequently,

$$
\begin{aligned}
\limsup _{t \rightarrow 1}\left|\partial_{t} f(t)\right| & =\limsup _{t \rightarrow 1}\left(1-t^{2}\right)^{-(n-1) / 2}\left|\int_{t}^{1} \frac{\mathrm{D}_{q} f(s)-q f(s)}{\left(1-s^{2}\right)^{1-(n-1) / 2}} d s\right| \\
& \leq \limsup _{t \rightarrow 1} \frac{1-t}{1-t^{2}}\left\|\mathrm{D}_{q} f-q f\right\|_{C^{0}} \leq C\left(\|f\|_{C^{0}}+\left\|\mathrm{D}_{q} f\right\|_{C^{0}}\right) .
\end{aligned}
$$

Similarly, we obtain $\lim \sup _{t \rightarrow-1}\left|\partial_{t} f(t)\right| \leq C\left(\|f\|_{C^{0}}+\left\|\mathrm{D}_{q} f\right\|_{C^{0}}\right)$ which, by (5.7), completes the proof of (5.4) and thus of (5.2) and (5.3). 
Next, assume that $q<0$. Then the maximum principle implies that there exists a $C>0$ such that

$$
\|f\|_{C^{0}} \leq C\left\|\mathrm{D}_{q} f\right\|_{C^{0}}
$$

for every $f \in C^{2}\left(\mathbb{S}^{n-1}\right)$. Consequently, we obtain from (5.3) that

$$
\|f\|_{C^{2}} \leq C\left\|\mathrm{D}_{q} f\right\|_{C^{0}}
$$

for every $\mathrm{SO}(n-1)$ invariant $f \in C^{2}\left(\mathbb{S}^{n-1}\right)$. From this, it follows that $\mathrm{D}_{q}$ is injective, has dense image and that

$$
\mathrm{D}_{q}^{-1}: C\left(\mathbb{S}^{n-1}\right)^{\mathrm{SO}(n-1)} \rightarrow C^{2}\left(\mathbb{S}^{n-1}\right)^{\mathrm{SO}(n-1)} \hookrightarrow C\left(\mathbb{S}^{n-1}\right)^{\mathrm{SO}(n-1)}
$$

exists and is bounded. Here, $C\left(\mathbb{S}^{n-1}\right)^{\mathrm{SO}(n-1)}$ denotes the Banach subspace of all $\mathrm{SO}(n-1)$ invariant functions in $C\left(\mathbb{S}^{n-1}\right)$ and $C^{2}\left(\mathbb{S}^{n-1}\right) \mathrm{SO}(n-1)$ is defined similarly. Moreover, the Arzelà-Ascoli theorem implies that $\mathrm{D}_{q}^{-1}$ is compact.

Now, choose an $m>n-1$ and put $q=n-m-1<0$. Applying the Fredholm alternative (see, e.g., [22, Theorem 5.3]) to the compact operator $\mathrm{D}_{q}^{-1}: C_{\mathrm{o}}\left(\mathbb{S}^{n-1}\right)^{\mathrm{SO}(n-1)} \rightarrow C_{\mathrm{o}}\left(\mathbb{S}^{n-1}\right)^{\mathrm{SO}(n-1)}$ yields that either

$$
f+m \mathrm{D}_{q}^{-1} f=0
$$

has a non-trivial solution $f \in C_{\mathrm{o}}\left(\mathbb{S}^{n-1}\right)^{\mathrm{SO}(n-1)}$ or

$$
f+m \mathrm{D}_{q}^{-1} f=\mathrm{D}_{q}^{-1} h
$$

has a solution for every $h \in C_{\mathrm{o}}\left(\mathbb{S}^{n-1}\right)^{\mathrm{SO}(n-1)}$. In the latter case, the operator $\left(\mathrm{Id}+m \mathrm{D}_{q}^{-1}\right)^{-1}$ is bounded. However, since

$$
\mathrm{D}_{q}\left(f+m \mathrm{D}_{q}^{-1} f\right)=\Delta_{\mathbb{S}} f+(n-1) f=0
$$

for $f \in C_{\mathrm{o}}\left(\mathbb{S}^{n-1}\right)^{\mathrm{SO}(n-1)}$ implies that $f=0$, equation (5.10) has no non-trivial solution in $C_{\mathrm{o}}\left(\mathbb{S}^{n-1}\right)^{\mathrm{SO}(n-1)}$ and thus (5.11) is solvable for every $h \in C_{\mathrm{o}}\left(\mathbb{S}^{n-1}\right)^{\mathrm{SO}(n-1)}$, that is, $h=\Delta_{\mathbb{S}} f+(n-1) f$ is solvable for every $h \in C_{\mathrm{o}}\left(\mathbb{S}^{n-1}\right)^{\mathrm{SO}(n-1)}$ and

$$
\|f\|_{C^{0}}=\left\|\left(\mathrm{Id}+m \mathrm{D}_{q}^{-1}\right)^{-1} \mathrm{D}_{q}^{-1} h\right\|_{C^{0}} \leq C\left\|\mathrm{D}_{q}^{-1} h\right\|_{C^{0}} \leq C\|h\|_{C^{0}}=C\left\|\mathrm{D}_{n-1} f\right\|_{C^{0}} .
$$

Combining this with (5.3) for the case $q=n-1$ and recalling that $\mathrm{D}_{n-1}=(n-1) \square_{n}$, completes the proof of the lemma.

We remark, that Lemma 5.2 without the assumption of $\mathrm{SO}(n-1)$ invariance does not hold in general.

Using Lemma 5.2 and Proposition 4.3, we can now complete the proof of Theorem 3 (b). 
Proof of Theorem 3 (b). Let $\phi_{i} \in \mathbf{V a l}_{i}^{\mathrm{SO}(n-1)}, 1 \leq i \leq n-1$, and recall that every $\mathrm{SO}(n-1)$ invariant valuation is spherical. Hence, using the Poincaré duality map, we can identify $\phi_{i}$ with a generalized valuation from $\mathbf{V a l}_{i}^{-\infty, \mathrm{sph}}$. By Proposition 4.3,

$$
\left\langle\phi_{i}, \mathrm{E}_{n-i} f\right\rangle=\frac{(n-i) !}{(n-1) !}\left\langle f \circ a, \nu_{\Lambda^{i-1} \phi_{i}}\right\rangle
$$

for $f \in C_{\mathrm{o}}^{\infty}\left(\mathbb{S}^{n-1}\right)$. Since $\Lambda^{i-1} \phi_{i}$ is 1-homogeneous, $\nu_{\Lambda^{i-1} \phi_{i}} \in C_{\mathrm{o}}^{-\infty}\left(\mathbb{S}^{n-1}\right)$ is of order at most 2 (cf. Example $3.1(\mathrm{~b}))$. Hence, $\phi_{i} \circ \mathrm{E}_{n-i} \in C_{\mathrm{o}}^{-\infty}\left(\mathbb{S}^{n-1}\right)$ defines an $\mathrm{SO}(n-1)$ invariant distribution of order at most 2 .

At the same time, by Theorem 5.1, $\phi_{i}=\widetilde{\mathrm{E}}_{i} \gamma$ for some uniquely determined $\gamma \in C_{\mathrm{o}}^{-\infty}\left(\mathbb{S}^{n-1}\right)$ and since $\phi_{i}$ is $\mathrm{SO}(n-1)$ invariant, so is $\gamma$. We want to show that $\gamma$ is of order 0 and, thus, in fact a measure. To this end, first note that, by (5.1),

$$
\phi_{i} \circ \mathrm{E}_{n-i}=\frac{(n-i) ! i !}{(n-1) !} \gamma \circ \square_{n} \circ a^{*} .
$$

Now, for $f \in C_{\mathrm{o}}^{\infty}\left(\mathbb{S}^{n-1}\right)$, let

$$
\bar{f}=\int_{\mathrm{SO}(n-1)} \vartheta f d \vartheta=\delta_{\bar{e}} * f
$$

denote the $\mathrm{SO}(n-1)$-rotational symmetral of $f$. Clearly, we have $\|\bar{f}\|_{C^{0}} \leq\|f\|_{C^{0}}$. Moreover, it is not difficult to show (cf. [53, Theorem 6.30]) that the $\mathrm{SO}(n-1)$ invariance of $\gamma$ implies $\gamma(f)=\gamma(\bar{f})$. Consequently, using Lemma 5.2, we obtain

$$
|\gamma(f)|=|\gamma(\bar{f})|=C\left|\left(\phi_{i} \circ \mathrm{E}_{n-i} \circ a^{*}\right)\left(\square_{n}^{-1} \bar{f}\right)\right| \leq C\left\|\square_{n}^{-1} \bar{f}\right\|_{C^{2}} \leq C\|\bar{f}\|_{C^{0}} \leq C\|f\|_{C^{0}},
$$

that is, $\gamma$ is of order 0 and therefore a measure.

In the case $i=n-1$, it follows from the result of McMullen [48, described in Example 3.1 (c), that, in fact, $\phi_{i} \in \widetilde{\mathrm{E}}_{i}\left(C_{\mathrm{o}}\left(\mathbb{S}^{n-1}\right)\right)$.

In the same way Theorem 4.1 implies Corollary 4.2, we can use Theorem 3 (b) and an approximation argument to deduce Theorem 2 .

Proof of Theorem 2. By Lemma 3.3, we have to show that for every $\operatorname{SO}(n)$ equivariant $F_{i} \in \mathbf{C V a l}_{i}, 1 \leq i \leq n-1$, there exist uniquely determined $\mathrm{SO}(n-1)$ invariant measures $\mu_{i} \in \mathcal{M}_{\mathrm{o}}\left(\mathbb{S}^{n-1}\right), 1 \leq i \leq n-2$, and an $\mathrm{SO}(n-1)$ invariant function $f_{n-1} \in C_{\mathrm{o}}\left(\mathbb{S}^{n-1}\right)$, such that for $1 \leq i \leq n-2$,

$$
F_{i, K}=S_{i}(K, \cdot) * \mu_{i}
$$

and

$$
F_{n-1, K}=S_{n-1}(K, \cdot) * f_{n-1}
$$

for every $K \in \mathcal{K}^{n}$. 
Since (5.13) can be proved, using Theorem 3 (b), in exactly the same way that (4.4) was deduced from Theorem 4.1, we only explain the proof of (5.12) here. First, let $K \in \mathcal{K}^{n}$ be such that $h_{K} \in C^{\infty}\left(\mathbb{S}^{n-1}\right)$ and that $K$ has positive curvature. Then the area measure $S_{i}(K, \cdot)$ of $K$ is absolutely continuous with respect to spherical Lebesgue measure with a smooth density function $s_{i}(K, \cdot) \in C_{\mathrm{o}}^{\infty}\left(\mathbb{S}^{n-1}\right)$ (see, e.g., [56, Chapter 2.5]). We want to show that if $\varphi_{i} \in \mathbf{V a l}_{i}^{\mathrm{sph}}$ denotes the $\mathrm{SO}(n-1)$ invariant associated real valued valuation of $F_{i}, 1 \leq i \leq n-2$, then there exists a uniquely determined $\mathrm{SO}(n-1)$ invariant $\mu_{i} \in \mathcal{M}_{\mathrm{o}}\left(\overrightarrow{\mathbb{S}^{n-1}}\right)$ such that

$$
\varphi_{i}(K)=\int_{\mathbb{S}^{n-1}} s_{i}(K, u) d \mu_{i}(u) .
$$

To this end, note that, by Theorem 3, there exists a uniquely determined $\mathrm{SO}(n-1)$ invariant $\mu_{i} \in \mathcal{M}_{\mathrm{o}}\left(\mathbb{S}^{n-1}\right)$ such that $\varphi_{i}=\widetilde{\mathrm{E}}_{i} \mu_{i}$. Moreover, it follows from a result of Bernig and Faifman [15, p. 11] that

$$
\varphi_{i}(K)=\left\langle\varphi_{i}, \psi_{n-i}^{K}\right\rangle,
$$

where $\psi_{n-i}^{K} \in \mathbf{V a l}_{n-i}^{\infty}$ is given by the mixed volume

$$
\psi_{n-i}^{K}(L)=\left(\begin{array}{c}
n \\
i
\end{array}\right) V(L[n-i],-K[i]) .
$$

Now, let $f_{i, j} \in C_{\mathrm{o}}^{\infty}\left(\mathbb{S}^{n-1}\right), j \in \mathbb{N}$, be a sequence of smooth functions which converges weakly to $\mu_{i}$. Then, by (5.15) and Proposition 4.3, we have

$$
\varphi_{i}(K)=\lim _{j \rightarrow \infty}\left\langle\widetilde{\mathrm{E}}_{i} f_{i, j}, \psi_{n-i}^{K}\right\rangle=\frac{i !}{(n-1) !} \lim _{j \rightarrow \infty}\left\langle f_{i, j} \circ a, \nu_{\Lambda^{n-i-1} \psi_{n-i}^{K}}\right\rangle .
$$

Using the definitions of $\psi_{n-i}^{K}$ and $\Lambda$ it is not difficult to show that

$$
\left(\Lambda^{n-i-1} \psi_{n-i}^{K}\right)(L)=\frac{n !}{i !} V(L, B[n-i-1],-K[i])=\frac{(n-1) !}{i !} \int_{\mathbb{S}^{n-1}} h_{L}(u) d S_{i}(-K, u) .
$$

Thus, using $d S_{i}(-K, u)=s_{i}(K,-u) d u$ and the definition of $\nu_{\Lambda^{n-i-1} \psi_{n-i}^{K}}$, we obtain

$$
\varphi_{i}(K)=\lim _{j \rightarrow \infty} \int_{\mathbb{S}^{n-1}} s_{i}(K, u) f_{i, j}(u) d u=\int_{\mathbb{S}^{n-1}} s_{i}(K, u) d \mu_{i}(u)
$$

which completes the proof of (5.14).

From the definition of $\varphi_{i},(5.14)$, and (2.17), we now obtain

$$
F_{i, K}(\bar{\eta})=\varphi_{i}\left(\eta^{-1} K\right)=\int_{\mathbb{S}^{n-1}} s_{i}(K, \eta u) d \mu_{i}(u)=\left(s_{i}(K, \cdot) * \mu_{i}\right)(\bar{\eta}) .
$$

Since both sides of this equation depend continuously on $K$, (5.12) follows from the fact that convex bodies with smooth support functions and positive curvature are dense in $\mathcal{K}^{n}$. 
The following consequence of Theorem 2 for homogeneous Minkowski valuations includes a slight improvement for dimensions $n \leq 4$ which we deduce from the existence of the derivation operator $\Lambda: \mathbf{M V a l} \rightarrow \mathbf{M V a l}$ and the estimate (3.5).

Corollary 5.3 Let $\Phi_{i}: \mathcal{K}^{n} \rightarrow \mathcal{K}^{n}$ be a continuous, translation invariant, and $\mathrm{SO}(n)$ equivariant Minkowski valuation of degree $i \in\{0, \ldots, n\}$.

(i) If $i=0$, then $\Phi_{0} K=c_{0} B$ for some $c_{0} \geq 0$ and every $K \in \mathcal{K}^{n}$.

(ii) If $1 \leq i \leq n-2$, then there exists a uniquely determined $\mathrm{SO}(n-1)$ invariant $\mu_{i} \in \mathcal{M}_{\mathrm{o}}\left(\mathbb{S}^{n-1}\right)$ such that $h_{\Phi_{i} K}=S_{i}(K, \cdot) * \mu_{i}$ for every $K \in \mathcal{K}^{n}$.

(iii) If $i=n-1$, then there exists a uniquely determined $\mathrm{SO}(n-1)$ invariant $f_{n-1} \in C_{\mathrm{o}}\left(\mathbb{S}^{n-1}\right)$ such that $h_{\Phi_{n-1} K}=S_{n-1}(K, \cdot) * f_{n-1}$ for every $K \in \mathcal{K}^{n}$.

(iv) If $i=n$, then $\Phi_{n} K=c_{n} V_{n}(K) B$ for some $c_{n} \geq 0$ and every $K \in \mathcal{K}^{n}$.

Moreover, if $n=3$ or $n=4$, then the measures $\mu_{i}, i=1,2$, from (ii) are absolutely continuous with respect to spherical Lebesgue measure with densities in $L_{\mathrm{o}}^{2}\left(\mathbb{S}^{n-1}\right)$.

Proof. The statements (i)-(iv) are direct consequences of Theorem 2, so we only have to prove the absolute continuity of the measures $\mu_{i}, i=1,2$, for $n \leq 4$. To this end, first note that $\Lambda^{i-1} \Phi_{i} \in \mathbf{M V a l}_{1}$ is $\mathrm{SO}(n)$ equivariant and that if $\varphi_{i} \in \mathbf{V a l}_{i}$ is the associated real valued valuation of $\Phi_{i}$, then $\Lambda^{i-1} \varphi_{i} \in \mathbf{V a l}_{1}$ is associated with $\Lambda^{i-1} \Phi_{i}$. Thus, it follows easily from (ii), (4.2), (2.21) and the fact that multiplier transformations commute that for every $K \in \mathcal{K}^{n}$,

$$
h_{\Lambda^{i-1} \Phi_{i} K}=i ! S_{1}(K, \cdot) * \mu_{i}=i ! h_{K} * \square_{n} \mu_{i} .
$$

Hence, the distribution determined by $\Lambda^{i-1} \varphi_{i} \in \mathbf{V a l}_{1}$ (cf. Example 3.1 (b)) is given by $i ! \square_{n} \mu_{i}$. Since $\mu_{i}$ is $\mathrm{SO}(n-1)$ invariant, so is $i ! \square_{n} \mu_{i}$ and it follows from (2.12) and (2.22) that the Fourier expansion of $i ! \square_{n} \mu_{i}$ is given by

$$
i ! \square_{n} \mu_{i} \sim i ! \sum_{k=0}^{\infty} \frac{N(n, k)}{\omega_{n}} \frac{(1-k)(k+n-1)}{n-1} a_{k}^{n}\left[\mu_{i}\right] P_{k}^{n}(\cdot \cdot \bar{e}) .
$$

Therefore, using (5.16), (2.18), and (3.5), it follows that there exists an absolute constant $C>0$ such that for every $k \geq 2$,

$$
\left|a_{k}^{n}\left[\mu_{i}\right]\right| \leq C \frac{i !(n-1)}{(k-1)(k+n-1)} .
$$

But, since $\left(\frac{N(n, k)}{\omega_{n}}\right)^{1 / 2} P_{k}^{n}(\cdot \cdot \bar{e})$ forms an orthonormal sequence in $L^{2}\left(\mathbb{S}^{n-1}\right)$ (see, e.g., [28, p. 84]) and, by (2.10), $N(n, k)=\mathrm{O}\left(k^{n-2}\right)$ as $k \rightarrow \infty$, we see that

$$
\mu_{i} \sim \sum_{k=0}^{\infty} \frac{N(n, k)}{\omega_{n}} a_{k}^{n}\left[\mu_{i}\right] P_{k}^{n}(\cdot \cdot \bar{e})
$$

converges in $L^{2}\left(\mathbb{S}^{n-1}\right)$ as long as $n \leq 4$. 
Corollary 5.3 (iii) was previously obtained by the first author [59] as already explained in Example 3.1 (c). The case $i=1$ of Corollary 5.3 (ii) can be reformulated as follows (cf. (5.16) ): There exists a uniquely determined $\mathrm{SO}(n-1)$ invariant $\mu_{1} \in \mathcal{M}_{\mathrm{o}}\left(\mathbb{S}^{n-1}\right)$ such that for every $K \in \mathcal{K}^{n}$,

$$
h_{\Phi_{1} K}=h_{K} * \square_{n} \mu_{1} .
$$

Comparing (5.18) with the corresponding result (3.3) of Kiderlen 36], shows that we have slightly improved (3.3) by proving that the $\mathrm{SO}(n-1)$ invariant distribution $\nu_{1} \in C_{\mathrm{o}}^{-\infty}\left(\mathbb{S}^{n-1}\right)$ of order at most 2 determined by $\Phi_{1}$ is always of the form $\nu_{1}=\square_{n} \mu_{1}$ for some $\mathrm{SO}(n-1)$ invariant $\mu_{1} \in \mathcal{M}_{\mathrm{o}}\left(\mathbb{S}^{n-1}\right)$.

Note that the estimate (5.17) is not strong enough to deduce that $\mu_{i}$ is absolutely continuous in higher dimensions, as can be seen for example from the spherical Radon (or Minkowski-Funk) transform, $\mathrm{R}: C\left(\mathbb{S}^{n-1}\right) \rightarrow C\left(\mathbb{S}^{n-1}\right)$, defined by

$$
\mathrm{R} f=f * \mu_{\mathbb{S} n-2},
$$

where $\mu_{\mathbb{S}^{n-2}} \in \mathcal{M}\left(\mathbb{S}^{n-1}\right)$ is uniformly concentrated on $\mathbb{S}^{n-1} \cap \bar{e}^{\perp}$. Clearly, $\mu_{\mathbb{S}^{n-2}}$ is $\mathrm{SO}(n-1)$ invariant but not absolutely continuous with respect to spherical Lebesgue measure. However, $\left|a_{k}^{n}\left[\mu_{\mathbb{S}^{n-2}}\right]\right|=\mathrm{O}\left(k^{1-n / 2}\right)$ as $k \rightarrow \infty$ (see, [28, Lemma 3.4.8]).

Finally we remark that Corollary 5.3 (ii) does not leave much room for improvement since the zonal functions $\breve{g}_{n}$ associated with Berg's functions are not continuous on $\mathbb{S}^{n-1}$ for $n \geq 3$ and they do not lie in $L_{\mathrm{o}}^{2}\left(\mathbb{S}^{n-1}\right)$ but merely in $L_{\mathrm{o}}^{1}\left(\mathbb{S}^{n-1}\right)$ for $n \geq 5$. However, they are generating functions of the normalized mean section operators $\mathrm{M}_{j}$ as described in Example 3.1 (d).

We conclude this section with an approximation result of continuous Minkowski valuations by smooth ones which generalizes a corresponding result for even Minkowski valuations of the first author 60. and will be useful in the last section.

Corollary 5.4 Every continuous translation invariant and $\mathrm{SO}(n)$ equivariant Minkowski valuation can be approximated uniformly on compact subsets of $\mathcal{K}^{n}$ by smooth translation invariant and $\mathrm{SO}(n)$ equivariant Minkowski valuations.

Proof. Let $\Phi \in \mathbf{M V a l}$ be $\mathrm{SO}(n)$ equivariant and let

$$
h_{\Phi K}=c_{0}+\sum_{i=1}^{n-2} S_{i}(K, \cdot) * \mu_{i}+S_{n-1}(K, \cdot) * f_{n-1}+c_{n} V_{n}(K)
$$

be the convolution representation of $\Phi$ according to Theorem 2. We define a sequence $\Phi^{j} \in \mathbf{M V a l}, j \in \mathbb{N}$, of $\operatorname{SO}(n)$ equivariant Minkowski valuations by

$$
h_{\Phi^{j} K}=h_{\Phi K} * h_{j}, \quad K \in \mathcal{K}^{n},
$$

where $h_{j}, m \in \mathbb{N}$, is a spherical approximate identity. Note that since $h_{j} \geq 0, \Phi^{j}$ is well defined by the result of Kiderlen [36] described at the end of Example 3.1 (b). Using (2.19) and the $\mathrm{SO}(n)$ equivariance of $\Phi$, it is easy to show that $\Phi^{j}$ converges to $\Phi$ on compact subsets (cf. the proof of [60. Theorem 6.5]). 
It remains to show that the Minkowski valuations $\Phi^{j}$ are smooth, that is, the associated real valued valuations $\varphi^{j} \in \mathbf{V a l}^{\text {sph }}$ are smooth. To this end note that by the linearity of the convolution, the fact that $\mu_{i} * h_{j}, f_{n-1} * h_{j} \in C^{\infty}\left(\mathbb{S}^{n-1}\right)$, and (2.17), we have

$\varphi^{j}(K)=c_{0}+\sum_{i=1}^{n-2} \int_{\mathbb{S}^{n-1}}\left(\mu_{i} * h_{j}\right)(u) d S_{i}(K, u)+\int_{\mathbb{S}^{n-1}}\left(f_{n-1} * h_{j}\right)(u) d S_{n-1}(K, u)+c_{n} V_{n}(K)$.

Thus, an application of Theorem 4.1 completes the proof.

\section{Integral Geometry of Minkowski Valuations}

In this final section we apply Theorem 2 to establish a Crofton formula for continuous, translation invariant, and $\mathrm{SO}(n)$ equivariant Minkowski valuations. Combining this with Hadwiger's general kinematic formula, allows us to also deduce a kinematic formula for such Minkowski valuations.

We begin by recalling the classical Crofton formula (see, e.g., [39, p. 124]) for intrinsic volumes: For $0 \leq i, j \leq n$ and $K \in \mathcal{K}^{n}$, we have

$$
\int_{\operatorname{AGr}_{n-i, n}} V_{j}(K \cap E) d \sigma_{n-i}(E)=\left[\begin{array}{c}
i+j \\
j
\end{array}\right] V_{i+j}(K)
$$

Here, $\mathrm{AGr}_{i, n}$ denotes the affine Grassmannian of $i$ planes in $\mathbb{R}^{n}$ and $\sigma_{i}$ is the rigid motion invariant measure on $\mathrm{AGr}_{i, n}$ normalized such that the set of planes having non-empty intersection with the Euclidean unit ball in $\mathbb{R}^{n}$ has measure

$$
\left[\begin{array}{c}
n \\
i
\end{array}\right] \kappa_{n-i}:=\left(\begin{array}{c}
n \\
i
\end{array}\right) \frac{\kappa_{n}}{\kappa_{i}} .
$$

The Crofton formula (6.1) is intimately related with the general kinematic formula: For $0 \leq j \leq n$ and $K, L \in \mathcal{K}^{n}$, we have

$$
\int \frac{V_{\mathrm{SO}(n)}}{j}(K \cap g L) d g=\sum_{i=0}^{n-j}\left[\begin{array}{c}
i+j \\
j
\end{array}\right]\left[\begin{array}{c}
n \\
i
\end{array}\right]^{-1} V_{i+j}(K) V_{n-i}(L),
$$

where $\overline{\mathrm{SO}(n)}=\mathrm{SO}(n) \ltimes \mathbb{R}^{n}$ (see the books [39, 58] for more information).

The obvious connection between (6.1) and (6.2) is just a special case of Hadwiger's general integral geometric theorem (see, [58, p. 173]), which states in the translation invariant case that for every $\phi \in \mathbf{V a l}$ and $K, L \in \mathcal{K}^{n}$, we have

$$
\int_{\frac{\mathrm{SO}(n)}{\phi}}(K \cap g L) d g=\sum_{i=0}^{n} V_{n-i}(L)\left[\begin{array}{c}
n \\
i
\end{array}\right]^{-1} \int_{\mathrm{AGr}_{n-i, n}} \phi(K \cap E) d \sigma_{n-i}(E) .
$$


An application of (6.3) to the real valued associated valuation of an $\mathrm{SO}(n)$ equivariant Minkowski valuation $\Phi \in$ MVal immediately yields the following kinematic formula for such Minkowski valuations.

Corollary 6.1 If $\Phi \in \mathbf{M V a l}$ is $\mathrm{SO}(n)$ equivariant, then

$$
\int_{\overline{\mathrm{SO}(n)}} h_{\Phi(K \cap g L)}(u) d g=\sum_{i=0}^{n} V_{n-i}(L)\left[\begin{array}{c}
n \\
i
\end{array}\right]^{-1} \int_{\mathrm{AGr}_{n-i, n}} h_{\Phi(K \cap E)}(u) d \sigma_{n-i}(E)
$$

for every $K, L \in \mathcal{K}^{n}$ and $u \in \mathbb{S}^{n-1}$.

Note that the sum on the right hand side of (6.4) is again the support function of a convex body. Thus, it remains to determine the Crofton integral in (6.4). In view of Lemma 3.3 and Theorem 2 , this is accomplished by our final result.

Theorem 6.2 Suppose that $1 \leq j \leq n-2$ and $1 \leq i \leq n-j-1$. If $F_{j} \in \mathbf{C V a l}_{i}$ is $\mathrm{SO}(n)$ equivariant and, for $K \in \mathcal{K}^{n}$, given by

$$
F_{j, K}=S_{j}(K, \cdot) * \mu
$$

for some (uniquely determined) $\mathrm{SO}(n-1)$ invariant measure $\mu \in \mathcal{M}_{\mathrm{o}}\left(\mathbb{S}^{n-1}\right)$, then

$$
\int_{\mathrm{AGr}_{n-i, n}} F_{j, K \cap E} d \sigma_{n-i}(E)=q_{n, i, j} S_{i+j}(K, \cdot) *\left(\mu * \square_{n-j+1} \breve{g}_{n-i-j+1}\right)
$$

where $q_{n, i, j}=\frac{2^{i}}{i ! \kappa_{i}} \prod_{k=j}^{i+j-1} c_{n, k}$ with

$$
c_{n, k}=\frac{k(n-k-1)(n-k+1) \kappa_{n-k-2}^{2} \kappa_{n-k+1} \kappa_{k}}{2(n-k)(k+1) \kappa_{n-k-3} \kappa_{n-k}^{2} \kappa_{k-1}} .
$$

Proof. Consider the isomorphism $\Theta_{j}: C_{\mathrm{o}}^{\infty}\left(\mathbb{S}^{n-1}\right) \rightarrow C_{\mathrm{o}}^{\infty}\left(\mathbb{S}^{n-1}\right)$, defined by

$$
\Theta_{j} f=c_{n, j} \square_{n-j+1} f * \breve{g}_{n-j}=c_{n, j} f * \square_{n-j+1} \breve{g}_{n-j} .
$$

Here and in (6.5),$\square_{k} \breve{g}_{l}$ is to be understood in the sense of distributions, where we use the canonical extension of the selfadjoint operator $\square_{k}$ to $C_{\mathrm{o}}^{-\infty}\left(\mathbb{S}^{n-1}\right)$.

Now, let us first assume that $F_{j}$ is smooth, that is, $\mu$ is absolutely continuous with respect to spherical Lebesgue measure with density $f \in C_{\mathrm{o}}^{\infty}\left(\mathbb{S}^{n-1}\right)$. In this case it was proved by the authors in [62, Theorem 6.3] that

$$
\int_{\operatorname{AGr}_{n-1, n}} F_{j, K \cap E} d \sigma_{n-1}(E)=S_{j+1}(K, \cdot) * \Theta_{j} f .
$$


In order to obtain from this the more general formula (6.5), we use the following well known relation (which can be proved by induction using Crofton's formula; see, e.g., [39, p. 124])

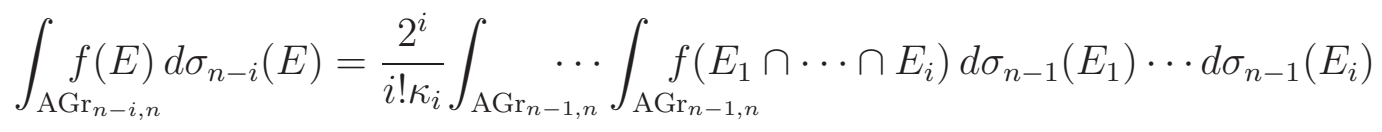

for every Borel measurable $f \in L^{1}\left(\mathrm{AGr}_{n-i, n}\right)$. Comparing this with (6.6), we obtain

$$
\int_{\operatorname{AGr}_{n-i, n}} F_{j, K \cap E} d \sigma_{n-i}(E)=\frac{2^{i}}{i ! \kappa_{i}} S_{i+j}(K, \cdot) * \Theta_{i+j-1} \cdots \Theta_{j+1} \Theta_{j} f .
$$

Next, note that if $\tau_{\bar{e}}=\delta_{\bar{e}}-\pi_{1} \delta_{\bar{e}} \in \mathcal{M}_{\mathrm{o}}\left(\mathbb{S}^{n-1}\right)$, where $\delta_{\bar{e}}$ is the Dirac measure supported in $\bar{e} \in \mathbb{S}^{n-1}$, then, by (2.18), $f * \tau_{\bar{e}}=f$ for every $f \in C_{\mathrm{o}}^{\infty}\left(\mathbb{S}^{n-1}\right)$. But since $\square_{k} \breve{g}_{k}=\tau_{\bar{e}}$, (6.5) follows from (6.7) and the definition of $\Theta_{j}$.

In order to establish (6.5) in the general case, where $F_{j}$ is merely continuous, we use a spherical approximate identity $h_{k}, k \in \mathbb{N}$, (instead of repeating the arguments from the proof of [62, Theorem 6.3]) to define $F_{j, K}^{k}=F_{j, K} * h_{k}$ for every $K \in \mathcal{K}^{n}$. Then, $F_{j}^{k} \in \mathbf{C V a l}_{j}$ is $\mathrm{SO}(n)$ equivariant and smooth and, by what we have already shown and the fact that multiplier transformations commute,

$$
\int_{\operatorname{AGr}_{n-i, n}} F_{j, K \cap E}^{k} d \sigma_{n-i}(E)=q_{n, i, j} S_{i+j}(K, \cdot) *\left(\mu * \square_{n-j+1} \breve{g}_{n-i-j+1}\right) * h_{k}
$$

Letting now $k \rightarrow \infty$, we obtain (6.5) from Lemmas 2.5 and 2.6 .

We conclude with the remark that equivalent forms of Theorem 6.2 were obtained very recently, independently, and using different approaches by Bernig and Hug [17] and Goodey, Hug, and Weil [26].

Acknowledgments The first author was supported by the European Research Council (ERC), Project number: 306445, and the Austrian Science Fund (FWF), Project number: Y603-N26. The second author was supported by the German Research Foundation (DFG), Project number: BE 2484/5-1.

\section{References}

[1] J. Abardia, Difference bodies in complex vector spaces, J. Funct. Anal. 263 (2012), 35883603 .

[2] J. Abardia and A. Bernig, Projection bodies in complex vector spaces, Adv. Math. 227 (2011), 830-846.

[3] S. Alesker, Continuous rotation invariant valuations on convex sets, Ann. of Math. (2) 149 (1999), 977-1005. 
[4] S. Alesker, Description of translation invariant valuations on convex sets with solution of $P$. McMullen's conjecture, Geom. Funct. Anal. 11 (2001), 244-272.

[5] S. Alesker, Hard Lefschetz theorem for valuations, complex integral geometry, and unitarily invariant valuations, J. Differential Geom. 63 (2003), 63-95.

[6] S. Alesker, The multiplicative structure on polynomial continuous valuations, Geom. Funct. Anal. 14 (2004), 1-26.

[7] S. Alesker, Hard Lefschetz theorem for valuations and related questions of integral geometry, Geometric aspects of functional analysis, 9-20, Lecture Notes in Math., 1850, Springer, Berlin, 2004.

[8] S. Alesker, A Fourier type transform on translation invariant valuations on convex sets, Israel J. Math. 181 (2011), 189-294.

[9] S. Alesker and D. Faifman, Convex valuations invariant under the Lorentz group, J. Differential Geom. 98 (2014), 183-236.

[10] S. Alesker, A. Bernig and F.E. Schuster, Harmonic analysis of translation invariant valuations, Geom. Funct. Anal. 21 (2011), 751-773.

[11] A. Berg, L. Parapatits, F.E.Schuster and M. Weberndorfer, Log-Concavity Properties of Minkowski Valuations, arXiv:1411.7891.

[12] C. Berg, Corps convexes et potentiels sphériques, Mat.-Fys. Medd. Danske Vid. Selsk. 37 (1969), 64 pp.

[13] A. Bernig, A Hadwiger-type theorem for the special unitary group, Geom. Funct. Anal. 19 (2009), 356-372.

[14] A. Bernig and L. Bröcker, Valuations on manifolds and Rumin cohomology, J. Differential Geom. 75 (2007), 433-457.

[15] A. Bernig and D. Faifman, Generalized translation invariant valuations and the polytope algebra, arXiv: 1406.4500

[16] A. Bernig and J.H.G. Fu, Hermitian integral geometry, Ann. of Math. (2) 173 (2011), 907945.

[17] A. Bernig and D. Hug, Kinematic formulas for tensor valuations, J. Reine Angew. Math., in press.

[18] J. Bernstein and B. Krötz, Smooth Fréchet globalizations of Harish-Chandra modules, Israel J. Math. 199 (2014), 45-111.

[19] T. Bröcker and T. tom Dieck, Representations of compact Lie groups. Graduate Texts in Mathematics 98, Springer-Verlag, New York, 1985.

[20] J.H.G. Fu, Structure of the unitary valuation algebra, J. Differential Geom. 72 (2006), 509533.

[21] R.J. Gardner, Geometric tomography, Second ed., Cambridge University Press, Cambridge, 2006.

[22] D. Gilbarg and N.S. Trudinger, Elliptic partial differential equations of second order, Reprint of the 1998 edition, Classics in Mathematics, Springer, Berlin, 2001.

[23] P. Goodey and W. Weil, Distributions and valuations, Proc. London Math. Soc. 49 (1984), 504-516.

[24] P. Goodey and W. Weil, The determination of convex bodies from the mean of random sections, Math. Proc. Cambridge Philos. Soc. 112 (1992), 419-430.

[25] P. Goodey and W. Weil, Sums of sections, surface area measures and the general Minkowski problem, J. Differential Geom. 97 (2014), 477-514.

[26] P. Goodey, D. Hug, and W. Weil, Kinematic formulas for area measures, arXiv:1507.03353. 
[27] E. Grinberg and G. Zhang, Convolutions, transforms, and convex bodies, Proc. London Math. Soc. (3) 78 (1999), 77-115.

[28] H. Groemer, Geometric Applications of Fourier Series and Spherical Harmonics, Cambridge University Press, Cambridge, 1996.

[29] V. Guillemin and S. Sternberg, Geometric asymptotics, Mathematical Surveys 14, American Mathematical Society, Providence, R.I., 1977.

[30] C. Haberl, Minkowski valuations intertwining the special linear group, J. Eur. Math. Soc. 14 (2012), 1565-1597.

[31] C. Haberl and L. Parapatits, The centro-affine Hadwiger theorem, J. Amer. Math. Soc. 27 (2014), 685-705.

[32] C. Haberl and F.E. Schuster, General $L_{p}$ affine isoperimetric inequalities, J. Differential Geom. 83 (2009), 1-26.

[33] H. Hadwiger, Vorlesungen über Inhalt, Oberfläche und Isoperimetrie, Springer, Berlin, 1957.

[34] L. Hörmander, The analysis of linear partial differential operators. I. Distribution theory and Fourier analysis, Fundamental Principles of Mathematical Sciences 256, Springer, Berlin, 1983.

[35] D. Hug, R. Schneider and R. Schuster, The space of isometry covariant tensor valuations, Algebra i Analiz 19 (2007), 194-224.

[36] M. Kiderlen, Blaschke- and Minkowski-Endomorphisms of convex bodies, Trans. Amer. Math. Soc. 358 (2006), 5539-5564.

[37] D.A. Klain, A short proof of Hadwiger's characterization theorem, Mathematika 42 (1995), 329-339.

[38] D.A. Klain, Even valuations on convex bodies, Trans. Amer. Math. Soc. 352 (2000), 71-93.

[39] D.A. Klain and G.-C. Rota, Introduction to geometric probability, Cambridge University Press, Cambridge, 1997.

[40] M. Ludwig, Projection bodies and valuations, Adv. Math. 172 (2002), 158-168.

[41] M. Ludwig, Minkowski valuations, Trans. Amer. Math. Soc. 357 (2005), 4191-4213.

[42] M. Ludwig, Minkowski areas and valuations, J. Differential Geom. 86 (2010), 133-161.

[43] M. Ludwig and M. Reitzner, A classification of SL( $n)$ invariant valuations, Ann. of Math. (2) 172 (2010), 1223-1271.

[44] E. Lutwak, D. Yang, and G. Zhang, $L_{p}$ affine isoperimetric inequalities, J. Differential Geom. 56 (2000), 111-132.

[45] E. Lutwak, D. Yang, and G. Zhang, Orlicz projection bodies, Adv. Math. 223 (2010), 220 242.

[46] E. Lutwak, D. Yang, and G. Zhang, Orlicz centroid bodies, J. Differential Geom. 84 (2010), $365-387$.

[47] P. McMullen, Valuations and Euler-type relations on certain classes of convex polytopes, Proc. London Math. Soc. 35 (1977), 113-135.

[48] P. McMullen, Continuous translation-invariant valuations on the space of compact convex sets, Arch. Math. 34 (1980), 377-384.

[49] M. Morimoto, Analytic functionals on the sphere, Translations of Mathematical Monographs 178, American Mathematical Society, Providence, RI, 1998.

[50] L. Parapatits and F.E. Schuster, The Steiner formula for Minkowski valuations, Adv. Math. 230 (2012), 978-994.

[51] L. Parapatits and T. Wannerer, On the inverse Klain map, Duke Math. J. 162 (2013), 1895-1922. 
[52] P. Petersen, Riemannian geometry, Second ed., Graduate Texts in Mathematics 171, Springer, New York, 2006.

[53] W. Rudin, Functional analysis, Second ed., International Series in Pure and Applied Mathematics, McGraw-Hill Inc., New York, 1991.

[54] R. Schneider, Equivariant endomorphisms of the space of convex bodies, Trans. Amer. Math. Soc. 194 (1974), 53-78.

[55] R. Schneider, Bewegungsäquivariante, additive und stetige Transformationen konvexer Bereiche, Arch. Math. 25 (1974), 303-312.

[56] R. Schneider, Convex Bodies: The Brunn-Minkowski Theory, Second ed., Encyclopedia of Mathematics and its Applications 151, Cambridge University Press, Cambridge, 2013.

[57] R. Schneider and F.E. Schuster, Rotation equivariant Minkowski valuations, Int. Math. Res. Not. (2006), Article ID 72894, 20 pages.

[58] R. Schneider and W. Weil, Stochastic and Integral Geometry, Probability and its Applications (New York), Springer, Berlin, 2008.

[59] F.E. Schuster, Convolutions and multiplier transformations of convex bodies, Trans. Amer. Math. Soc. 359 (2007), 5567-5591.

[60] F.E. Schuster, Crofton measures and Minkowski valuations, Duke Math. J. 154 (2010), 1-30.

[61] F.E. Schuster and T. Wannerer, GL $(n)$ contravariant Minkowski valuations, Trans. Amer. Math. Soc. 364 (2012), 815-826.

[62] F.E. Schuster and T. Wannerer, Even Minkowski valuations, Amer. J. Math., in press.

[63] W. Spiegel, Zur Minkowski-Additivität bestimmter Eikörperabbildungen, J. Reine Angew. Math. 286/287 (1976), 164-168.

[64] M. Takeuchi, Modern spherical functions, Transl. Math. Monogr. 135, Amer. Math. Soc., Providence, RI, 1994.

[65] T. Wannerer, GL(n) equivariant Minkowski valuations, Indiana Univ. Math. J. 60 (2011), $1655-1672$

[66] T. Wannerer, The module of unitarily invariant area measures, J. Differential Geom. 96 (2014), 141-182.

[67] T. Wannerer, Integral geometry of unitary area measures, Adv. Math. 263 (2014), 1-44.

[68] G. Warner, Harmonic analysis on semi-simple Lie groups I, Springer, Berlin, 1972.

Franz Schuster

Vienna University of Technology

franz.schuster@tuwien.ac.at
Thomas Wannerer

Goethe University Frankfurt

wannerer@mathematik.uni-frankfurt.de 